\title{
DEVELOPMENT AND PRELIMINARY ASSESSMENT OF A THREE-DIMENSIONAL THERMAL HYDRAULICS CODE, CUPID
}

\author{
JAE JUN JEONG*, HAN YOUNG YOON, IK KYU PARK, HYOUNG KYU CHO and HEEDONG LEE ${ }^{1}$ \\ Korea Atomic Energy Research Institute (KAERI) \\ 1045 Daeduk-daero, Yuseong-gu, Daejeon 305-353, Korea \\ ${ }^{1}$ Department of Aerospace Engineering, Korea Advanced Institute of Science and Technology (KAIST) \\ 373-1, Yuseong-gu, Daejeon, Korea \\ "Corresponding author. E-mail : jjjeong@kaeri.re.kr
}

Received March 09, 2010

Accepted for Publication April 21, 2010

For the analysis of transient two-phase flows in nuclear reactor components, a three-dimensional thermal hydraulics code, named CUPID, has been developed. The CUPID code adopts a two-fluid, three-field model for two-phase flows, and the governing equations were solved over unstructured grids, which are very useful for the analysis of flows in complicated geometries. To obtain numerical solutions, the semi-implicit numerical method for the REALP5 code was modified for an application to unstructured grids, and it has been further improved for enhanced accuracy and fast running. For the verification of the CUPID code, a set of conceptual problems and experiments were simulated. This paper presents the flow model, the numerical solution method, and the results of the preliminary assessment.

KEYWORDS : Multi-dimensional Two-phase Flow, Transient Analysis, Two-fluid Three-field Model, Unstructured Grids, Verification, The CUPID Code

\section{INTRODUCTION}

A realistic simulation of two-phase flows is essential for the advanced design and safe operation of a nuclear reactor system. The need for a multi-dimensional analysis of thermal-hydraulics in nuclear reactor components is further increasing with advanced design features, such as direct vessel injection systems, gravity-driven safety injection systems, and passive secondary cooling systems. Because of the complicated multi-dimensional flow effects and/or the reduced driving force in comparison with conventional active systems, more detailed analysis with enhanced accuracy is required. The use of computational fluid dynamics (CFD) codes for a more detailed analysis of two-phase flows has been studied extensively and such codes are known to be very promising [1-4]. But the modeling capabilities of most of the current CFD codes are limited to certain two-phase flow regimes and, furthermore, the use of these codes requires a huge computational cost [5]. Thus, the direct use of two-phase CFD codes for system transient analysis is not likely to be practical for a few decades. In this regard, the concept of a "multi-scale" analysis has been developed; this system aims at advanced simulation by adopting the combined use of different scale computational tools, such as system codes, component codes, CFD codes, and the smallest possible scale codes [6,7].

KAERI has developed a three-dimensional thermal hydraulics code, CUPID [8-12], for the analysis of transient, multi-dimensional, two-phase flows in nuclear reactor components. The code was designed for use as a componentscale code, and/or a three-dimensional component, which can be coupled with a system code. In the CUPID code, a two-fluid three-field model is adopted for two-phase flows, and the governing equations are solved on unstructured grids, which are very useful for the analysis of flows in complicated geometries. In two-phase momentum equations, non-drag forces [10], such as lift, wall lubrication, and turbulent dispersion forces are modeled in addition to the interfacial drag forces. These features distinguish the CUPID code from the three-dimensional components of system codes such as RELAP5-3D [13] and CATHARE 2 [14]. The physical modeling and the numerical solution method of the CUPID code put emphasis on versatile and robust simulations of complicated two-phase flows. This is based on a practical need to overcome the shortcomings of existing CFD codes and the three-dimensional component of system codes. For example, downcomer boiling during the reflood phase of a large-break, loss-of-coolant accident has been an important issue in the advanced reactor development [15]. It is characterized by the combined 
effects of boiling due to heat transfer from the downcomer wall, multi-dimensional counter-current flow, lateral motion of bubbles, flow regime changes, bulk condensation, and phase separation. The system codes, such as RELAP5/MOD3 and MARS [16], were not applicable to the realistic analysis of the phenomena, and some commercial CFD codes failed to yield a converged solution. Instead, the issue was addressed through experimental research.

This paper presents an overview of the CUPID code development and preliminary assessment, mainly focusing on the numerical solution method and its verification. In Section 2, the governing equations and closure relations for two-phase flows are presented. Section 3 gives the numerical solution method, which includes the unique features of the CUPID code such as the improved numerical scheme for fast running, second-order upwind scheme for the convective terms, and a new scheme to calculate the pressure gradient on unstructured grids. Section 4 presents the results of the preliminary assessment. The heat conduction model also plays an important role in the CUPID code. Description of the heat conduction model, however, is skipped here. It should also be noted that the preliminary assessment in Section 4 puts emphasis on the verification of the numerical solution method rather than on the validation.

\section{GOVERNING EQUATIONS AND CLOSURE RELATIONS FOR TWO-PHASE FLOWS}

To describe two-phase flows, a transient two-fluid three-field model is adopted in the CUPID code. The three fields represent a continuous liquid, an entrained liquid, and a vapor field [16,17]. The three-field model is particularly useful for modeling the thermal-hydraulics of a reactor core and containment during a hypothetical large-break loss of coolant accident. The CUPID governing equations take into account the porosity concept to model the two-phase flows at the component scale. However, for simplicity, the concept is omitted in this paper.

\subsection{The Governing Equations of the Two-Fluid Three-Field Model}

At first, the mass, energy, and momentum equations for each field are established separately and, then, they are linked by the interfacial mass, energy and momentum transfer models. The continuity equation for $\mathrm{k}$-field is

$$
\frac{\partial}{\partial t}\left(\alpha_{k} \rho_{k}\right)+\nabla \cdot\left(\alpha_{k} \rho_{k} \underline{u}_{k}\right)=\Omega_{k}
$$

where

$$
\begin{aligned}
& \Omega_{v}=\Gamma_{v}, \\
& \Omega_{l}=-(1-\eta) \Gamma_{v}-S_{E}+S_{D E}, \\
& \Omega_{d}=-\eta \Gamma_{v}+S_{E}-S_{D E}, \\
& \eta=\alpha_{d} /\left(\alpha_{l}+\alpha_{d}\right) .
\end{aligned}
$$

The non-condensable gases, when present, are contained in the vapor field, and these are assumed to move with the same velocity and have the same temperature as the vapor phase. Thus, the continuity equation for the total noncondensable component is given as

$$
\frac{\partial}{\partial t}\left(\alpha_{v} \rho_{v} X_{n}\right)+\nabla \cdot\left(\alpha_{v} \rho_{v} X_{n} \underline{u}_{v}\right)=0
$$

where the noncondensable gas quality $X_{n}$ is defined as the ratio of the noncondensable gas mass to the total gaseous phase mass.

The momentum equation for $\mathrm{k}$-field is:

$$
\begin{aligned}
& \frac{\partial}{\partial t}\left(\alpha_{k} \rho_{k} \underline{u}_{k}\right)+\nabla \cdot\left(\alpha_{k} \rho_{k} \underline{u}_{k} \underline{u}_{k}\right)= \\
& -\alpha_{k} \nabla P+\nabla \cdot\left[\alpha_{k}\left(\tau_{k}+\tau_{k}^{T}\right)\right]+\alpha_{k} \rho_{k} \underline{g}+\underline{M}_{i k}+\underline{M}_{k}^{N D},
\end{aligned}
$$

where $\underline{M}_{i k}$ is the interfacial momentum transfer due to interfacial drag, virtual mass, and interfacial mass transfer. $\underline{M}_{k}^{N D}$ is the interfacial momentum transfer terms due to non-drag forces. The turbulent viscosity is obtained by using the standard k- $\varepsilon$ model or the mixing length model.

For the energy equations, it is assumed that the continuous liquid and the entrained liquid are in a thermal equilibrium, i.e., $T_{d}=T_{l}$ and $\rho_{d}=\rho_{l}$ [16]. As a result, only two energy equations are used, i.e., one for the vapor field and one for the combined liquid field:

$$
\begin{aligned}
& \frac{\partial}{\partial t}\left(\alpha_{v} \rho_{v} e_{v}\right)+\nabla \cdot\left(\alpha_{v} \rho_{v} e_{v} \underline{u}_{v}\right) \\
& =E_{v}^{D}-P \frac{\partial}{\partial t} \alpha_{v}-P \nabla \cdot\left(\alpha_{v} \underline{u}_{v}\right)+Q_{i v}-Q_{v l}, \\
& \frac{\partial}{\partial t}\left[\left(1-\alpha_{v}\right) \rho_{l} e_{l}\right]+\nabla \cdot\left[\left(\alpha_{l} \underline{u}_{l}+\alpha_{d} \underline{u}_{d}\right) \rho_{l} e_{l}\right] \\
& =E_{l}^{D}-P \frac{\partial}{\partial t}\left(1-\alpha_{v}\right)-P \nabla \cdot\left(\alpha_{l} \underline{u}_{l}+\alpha_{d} \underline{u}_{d}\right)+Q_{i l}+Q_{v l},
\end{aligned}
$$

where $E_{k}^{D}$ includes the k-phase conduction, turbulent energy source, and viscous dissipation.

\subsection{Interfacial Heat, Mass, and Momentum Transfers}

The interfacial energy transfer terms, $Q_{i v}$ and $Q_{i l}$, in Eqs. (4) and (5), are modeled as:

$$
\begin{gathered}
\left.Q_{i v}=\frac{P_{s}}{P} H_{i v}\left[T_{s a t}\left(P_{s}\right)-T_{v}\right)\right]+\Gamma_{v} h_{v i}, \\
Q_{i l}=H_{i l}\left[T_{s a t}\left(P_{s}\right)-T_{l}\right]-\Gamma_{v} h_{l i},
\end{gathered}
$$

where the first terms in the right-hand side of Eqs. (6) and (7) are the bulk interface heat transfer and the second terms are the interface energy transfer due to interface mass transfer. $P_{s}$ is the steam partial pressure. The interface 
enthalpies $h_{v i}$ and $h_{l i}$ are defined in such a way that the interface energy jump conditions at the liquid-vapor interface are satisfied [13]. Because the sum of $Q_{i v}$ and $Q_{i l}$ is zero, the volumetric vapor generation rate is represented as:

$$
\Gamma_{v}=-\frac{\left.\left.\frac{P_{s}}{P} H_{i v}\left[T_{s a t}\left(P_{s}\right)-T_{v}\right)\right]+H_{i l}\left[T_{s a t}\left(P_{s}\right)-T_{l}\right)\right]}{h_{v i}-h_{l i}} .
$$

The term $Q_{v l}$ in Eqs. (4) and (5) is the sensible heat transfer rate per unit volume at the noncondensable gasliquid interface:

$$
\begin{aligned}
Q_{v l} & =\frac{P-P_{s}}{P} H_{v l}\left(T_{v}-T_{l}\right) \\
& =\frac{P_{n}}{P} H_{v l}\left(T_{v}-T_{l}\right),
\end{aligned}
$$

where $H_{v l}$ is the sensible heat transfer coefficient per unit volume and $P_{n}$ is the noncondensable gas partial pressure. The multiplier based on the pressures is an ad-hoc function used to turn off this term when there is no noncondensable gas [13].

The interfacial momentum transfer term, $\underline{M}_{i k}$ in Eq. (3), includes the interfacial drag, the momentum exchange due to the interface mass transfer, and virtual mass force. For simplicity, the virtual mass term is omitted hereafter and $M_{i k}$ is written as:

$$
\begin{aligned}
& \underline{M}_{i v}=F_{v l}\left(\underline{u}_{l}-\underline{u}_{v}\right)+F_{v d}\left(\underline{u}_{d}-\underline{u}_{v}\right)+\Gamma_{v} \underline{u}_{v i}, \\
& \underline{M}_{i l}=F_{v l}\left(\underline{u}_{v}-\underline{u}_{l}\right)-(1-\eta) \Gamma_{v} \underline{u}_{l i}-S_{E} \underline{u}_{l}+S_{D E} \underline{u}_{d}, \\
& \underline{M}_{i d}=F_{v d}\left(\underline{u}_{v}-\underline{u}_{d}\right)-\eta \Gamma_{v} \underline{u}_{d i}+S_{E} \underline{u}_{l}-S_{D E} \underline{u}_{d},
\end{aligned}
$$

where $F$ 's in the right-hand side of Eqs. (10) through (12) are the coefficients for the interfacial drag forces. The interface velocities, $\underline{\boldsymbol{u}}_{k}$, are needed to obtain the interfacial momentum transfer due to the interface mass transfer. These are determined using a donor formulation concept [13]. The non-drag force term, $M_{k}^{N D}$ in Eq. (3), represents the forces acting perpendicular to the mean flow direction, including the lift, wall lubrication, and turbulent dispersion terms [10].

\subsection{Constitutive Relationships and Equations of the State (EOS)}

For mathematical closure, the undefined terms and coefficients in the right-hand side of the governing equations should be given as functions of the independent state variables and phasic velocities. These require physical models for interfacial area concentration, interfacial heat and momentum transfer, non-drag forces, droplet entrainment and deposition, and wall heat transfer model. Because all these are dependent on a two-phase flow regime, they again require some relevant models. All these constitutive relations of the CUPID code [10,12] were adopted from the state-of-the-art two-phase flow codes.

Equations of the states are also needed. In the CUPID code, the phasic density and temperature of the liquid field are expressed as functions of the pressure and internal energy:

$$
\begin{gathered}
\rho_{l}=\rho_{l}\left(P, e_{l}\right), \\
T_{l}=T_{l}\left(P, e_{l}\right) .
\end{gathered}
$$

The properties for the gaseous phase are calculated assuming a modified Gibbs-Dalton mixture of steam and an ideal noncondensable gas:

$$
\begin{gathered}
\rho_{v}=\rho_{v}\left(P, e_{v}, X_{n}\right), \\
T_{v}=T_{v}\left(P, e_{v}, X_{n}\right), \\
P_{s}=P_{s}\left(P, e_{v}, X_{n}\right) .
\end{gathered}
$$

The saturation property is represented as a function of the pressure:

$$
T_{\text {sat }}=T_{\text {sat }}\left(P_{s}\right)
$$

\section{NUMERICAL SOLUTION METHOD}

In this section, the basic CUPID numerical solution method [8] is described first. Then, the unique features of the CUPID code are presented, such as the improved numerical scheme for fast running, the second-order upwind scheme for the convective terms, and a new scheme to obtain the pressure gradient.

\subsection{A Semi-Implicit Numerical Solution Scheme}

In the CUPID code, the finite volume method is applied to the integration of the governing equations on unstructured grids. All of the system variables of pressure, velocities, volume fractions, and energies are defined at a cell center. The cell-faced values are interpolated using the cellcentered values. Figure 1 shows an example of an unstructured grid in two dimensions, where $j$ is the neighboring cells of cell $i, f$ is the face number of cell $i$, and $\underline{S}_{f}$ is the area vector of the face $f$ between cell $i$ and $j$.

The solution algorithm of the CUPID code is based 


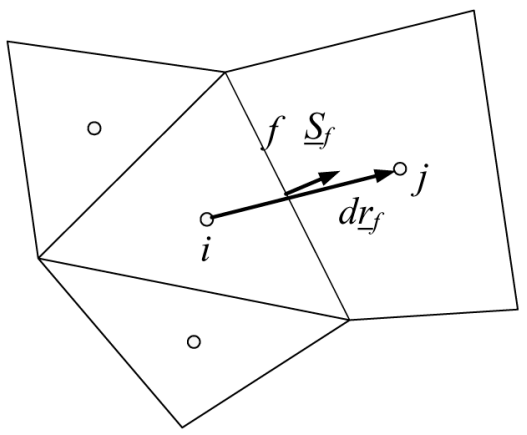

Fig. 1. Control Volumes and Geometric Vectors for a Finite Volume Discretization

on the semi-implicit method of the RELAP5 code $[13,18]$ with some modifications for application to unstructured non-staggered grids. In the RELAP5 code, the momentum equations in Eq. (3) are solved in the non-conservative form for numerical convenience as follows:

$$
\begin{aligned}
& \alpha_{k} \rho_{k} \frac{\partial \vec{u}_{k}}{\partial t}+\alpha_{k} \rho_{k} \vec{u}_{k} \cdot \nabla \vec{u}_{k}+\vec{u}_{k} \Omega_{k} \\
& =-\alpha_{k} \nabla P+\nabla \cdot\left[\alpha_{k}\left(\tau_{k}+\tau_{k}^{T}\right)\right]+\alpha_{k} \rho_{k} \underline{g}+\underline{M}_{i k}+\underline{M}_{k}^{N D} .
\end{aligned}
$$

However, in the CUPID code, a semi-conservative form is used, which is closer to the conservative form but still maintains the numerically convenient feature of the nonconservative form:

$$
\begin{aligned}
& \alpha_{k} \rho_{k} \frac{\partial \vec{u}_{k}}{\partial t}+\nabla \cdot\left(\alpha_{k} \rho_{k} \vec{u}_{k} \vec{u}_{k}\right)-\vec{u}_{k} \nabla \cdot\left(\alpha_{k} \rho_{k} \vec{u}_{k}\right)+\vec{u}_{k} \Omega_{k} \\
& =-\alpha_{k} \nabla P+\nabla \cdot\left[\alpha_{k}\left(\tau_{k}+\tau_{k}^{T}\right)\right]+\alpha_{k} \rho_{k} \underline{g}+\underline{M}_{i k}+\underline{M}_{k}^{N D} .
\end{aligned}
$$

The semi-conservative form in Eq. (20) is still nonconservative in time but conservative in space. Eq. (20) reduces to Eq. (3) in a steady state; in other words, the semi-conservative form becomes a conservative form in the case of a steady state. The numerical advantages of this method were presented by Park et al. [9].

To obtain the numerical solution of the two-fluid equations, at first, the momentum equation, Eq. (20), is integrated over a cell $i$, as shown in Fig. $1 . M_{i k}$ and the pressure gradient term in Eq. (20) are treated implicitly, whereas the convection and diffusion terms are calculated explicitly. Then, the phasic momentum equations are spatially not linked and we can obtain three simultaneous linear equations with three unknown phasic velocities, $\underline{u}_{k, i}^{*}$. The superscript $*$ indicates a temporal value. After solving the phasic momentum equations, we obtain the temporal phasic velocity represented by

$$
\underline{u}_{k, i}^{*}=\underline{\gamma}_{k, i}^{n}+\beta_{k, i} \nabla P_{i}^{n}
$$

where $\gamma_{k, i}^{n}$ includes the explicit convection, diffusion, and body force contributions and $\beta_{k, i}$ is the coefficient of the pressure gradient. The new velocity is implicitly linked to a new pressure from the momentum equation as given by:

$$
\underline{u}_{k, i}^{n+1}=\underline{\gamma}_{k, i}^{n}+\beta_{k, i} \nabla P_{i}^{n+1}
$$

Subtracting Eq. (21) from Eq. (22) gives:

$$
\underline{u}_{k, i}^{n+1}=\underline{u}_{k, i}^{*}+\beta_{k, i} \nabla P_{i}^{\prime},
$$

where $P_{i}^{\prime}\left(=P_{i}{ }^{n+1}-P_{i}{ }^{n}\right)$ is a pressure correction that will be determined from the coupled mass and energy equations.

Next, the six scalar equations, Eqs. (1), (2), (4), and (5), are integrated over the cell $i$. In this step, the convection terms are treated by using implicit velocities, but the convective quantities, such as the volume fraction, density, and internal energy are treated explicitly. The interfacial heat and mass transfer terms are treated implicitly. For the non-linear terms, first-order Taylor series expansions at the old time steps are used to linearly obtain variables at the new time step. For example, the vapor continuity equation is discretized and linearized as follows:

$$
\begin{aligned}
& \left(\rho_{v} \alpha_{v}^{\prime}+\alpha_{v} \rho_{v}^{\prime}\right)_{i} \frac{V_{i}}{\Delta t}+\sum_{f}\left(\alpha_{v} \rho_{v}\right)_{f} \underline{u}_{v, f}^{n+1} \cdot \underline{S}_{f}= \\
& -\frac{V_{i}}{h_{v}^{*}-h_{l}^{*}}\left[\frac{P_{s}}{P} H_{i v}\left(T_{s a t}^{n+1}-T_{v}^{n+1}\right)+H_{i l}\left(T_{s a t}^{n+1}-T_{l}^{n+1}\right)\right]_{i},
\end{aligned}
$$

where the new time temperatures are linearized as:

$$
\begin{aligned}
& T_{\text {sat }}^{n+1}=T_{\text {sat }}^{n}+\frac{\partial T_{\text {sat }}}{\partial P} P^{\prime}+\frac{\partial T_{\text {sat }}}{\partial e_{v}} e_{v}^{\prime}+\frac{\partial T_{\text {sat }}}{\partial X_{n}} X_{n}^{\prime}, \\
& T_{v}^{n+1}=T_{v}^{n}+\frac{\partial T_{v}}{\partial P} P^{\prime}+\frac{\partial T_{v}}{\partial e_{v}} e_{v}^{\prime}+\frac{\partial T_{v}}{\partial X_{n}} X_{n}^{\prime}, \\
& T_{l}^{n+1}=T_{l}^{n}+\frac{\partial T_{l}}{\partial P} P^{\prime}+\frac{\partial T_{l}}{\partial e_{l}} e_{l}^{\prime} .
\end{aligned}
$$

This is repeated for other scalar equations and the resulting linear equations are ordered in the following sequence: (i) the noncondensable gas continuity equation, (ii) the vapor energy equation, (iii) the total liquid energy equation, (iv) the vapor continuity equation, (v) the entrained liquid continuity equation, and (vi) the continuous liquid continuity equation. These linearized equations are rearranged with respect to the six independent scalar variables $\left(X_{n}^{\prime}, e^{\prime}{ }_{v}, e_{l}^{\prime}, \alpha_{v}^{\prime}, \alpha_{d}^{\prime}, P^{\prime}\right)$ as:

$$
\underline{A}_{i} \underline{x}_{i}=\underline{s}_{i}+\sum_{f} \sum_{k} \underline{c}_{k, f} \Psi_{k, f}^{n+1}
$$

where $\underline{\underline{A}}_{i}$ is a $6 \times 6$ coefficient matrix obtained from the 
above-mentioned six scalar equations,

$\underline{x}_{i}$ : the solution vector, $\underline{x}_{i}=\operatorname{col}\left(X_{n}^{\prime}, e_{v}^{\prime}, e_{l}^{\prime}, \alpha_{v}^{\prime}, \alpha_{d}^{\prime}, P^{\prime}\right)_{i}$,

$\Psi_{k, f}^{n+1}$ : phasic volume flow at the cell face $i j, \Psi_{k, f}^{n+1}=$ $\underline{u}_{k, f}^{n+1} \cdot \underline{S}_{f}$

$\underline{s}_{i}$ and $\underline{c}_{k, f}:$ coefficient vectors (known).

For instance, the coefficient vectors for the vapor-phase continuity equation are given by

$$
\begin{aligned}
& \underline{c}_{v, f}=\left(\alpha_{v} \rho_{v}\right)_{f}, \\
& \underline{c}_{l, f}=0, \\
& \underline{c}_{d, f}=0, \\
& \underline{S}_{i}=-\frac{V_{i}}{h_{v}^{*}-h_{l}^{*}}\left[\frac{P_{s}}{P} H_{i v}\left(T_{s a t}^{n}-T_{v}^{n}\right)+H_{i l}\left(T_{s a t}^{n}-T_{l}^{n}\right)\right]_{i} .
\end{aligned}
$$

The solution vector is obtained by multiplying Eq. (25) by $\underline{\underline{A}}_{i}^{-1}$.

$$
\underline{x}_{i}=\underline{\underline{A}}_{i}^{-1} \underline{S}_{i}+\sum_{f} \underline{\underline{A}}_{i}^{-1} \sum_{k} \underline{c}_{k, f} \Psi_{k, f}^{n+1} .
$$

The volume flow of $\Psi_{k, f}^{n+1}$ is obtained by applying a divergence operator of $\nabla$. to Eq. (23) and integrating it over the computing cell $i$ :

$$
\sum_{f} \Psi_{k, f}^{n+1}=\sum_{f}\left[\Psi_{k, f}^{*}+\beta_{k, f} \frac{A_{f}}{\left|\underline{d r_{f}}\right|}\left(P_{j}^{\prime}-P_{i}^{\prime}\right)\right] .
$$

Substituting Eq. (27) into the sixth row of Eq. (26) yields a pressure correction equation of

$$
\left(1+\sum_{f} C_{f}\right) P^{\prime}{ }_{i}-\sum_{f} C_{f} P^{\prime}{ }_{j}=B_{i}
$$

where

$$
\begin{aligned}
C_{f} & =\frac{A_{j}}{|\underline{d \underline{r}} f|} \sum_{k}\left(\underline{\underline{A}}_{i}^{-1} \underline{c}_{k, f}\right)_{6} \beta_{k, f}, \\
B_{i} & =\left(\underline{\underline{A}}_{i}^{-1} \underline{S}_{i}\right)_{6}+\sum_{f} \sum_{k}\left(\underline{\underline{A}}_{i}^{-1} \underline{c}_{k, f}\right)_{6} \Psi_{k, f}^{*} .
\end{aligned}
$$

By repeating this procedure, we can establish an $\mathrm{N} \times \mathrm{N}$ system of linear equations for the new time pressures for $\mathrm{N}$ computing cells, resulting in a non-symmetric matrix. An iterative method like the conjugate gradient method could be used to get a solution from the linear equation. After solving Eq. (28), the new time phasic velocity $\underline{u}_{k, i}^{n+l}$ and volume flow $\Psi_{k, f}^{n+1}$ are determined from Eqs. (23) and (27), respectively. Finally, the scalar variables are updated from Eq. (26) and the remaining state variables, such as density and temperature, are calculated using the EOS.

One can show that the interpolation of a new time phasic velocity at a cell face [19] is identical to the Rhie-
Chow scheme [20].

$$
\underline{u}_{k, f}^{n+1}=F\left(\underline{u}_{k, i}^{n+1}, \underline{u}_{k, j}^{n+1}\right)-F\left(\beta_{k, i}, \beta_{k, j}\right)\left\{\frac{P_{i}^{\prime}-P_{j}^{\prime}}{\left|\underline{d r_{f}}\right|} \underline{n}_{f}-F\left(\nabla P_{i}^{\prime}, \nabla P^{\prime}{ }_{j}\right)\right\}
$$

where $F\left(\varphi_{i}, \varphi_{j}\right)$ is a linear interpolation function with a distance weighting factor of $\xi$ :

$$
F\left(\varphi_{i}, \varphi_{j}\right)=\xi \varphi_{j}+(1-\xi) \varphi_{i} .
$$

\subsection{An Improved Numerical Solution Scheme for Fast Running}

The asymmetric system matrix of Eq. (28) requires long computational time to solve and it is sometimes hard to get the converged solution, especially when the number of cells is very large. In the new numerical scheme, the pressure calculation procedure is modified so that the pressure matrix becomes symmetric. Combining the mass conservation equations of Eqs. (1), (2), and (3), we obtain:

$$
\sum_{k} \frac{1}{\rho_{k}}\left(\nabla \cdot \alpha_{k} \rho_{k} \underline{u}_{k}\right)=\sum_{k}\left(\frac{\Omega_{k}}{\rho_{k}}-\frac{\alpha_{k}}{\rho_{k}} \frac{\partial \rho_{k}}{\partial t}\right) .
$$

Equation (31) is integrated over the cell $i$ in the same way as described in Section 3.1:

$$
\sum_{k} \frac{1}{\rho_{k}}\left(\nabla \cdot \alpha_{k} \rho_{k} \underline{u}_{k}^{n+1}\right)_{i}=\sum_{k}\left(\frac{\Omega_{k}^{n+1}}{\rho_{k}}-\frac{\alpha_{k}}{\rho_{k}} \frac{\rho_{k}^{\prime}}{\Delta t}\right)_{i}
$$

where variables without superscript are the old values. Thereafter, Equation (23) is substituted into Eq. (32) to eliminate the new time velocities. Two unknowns in the right-hand side of Eq. (32) are represented by

$$
\begin{gathered}
\Omega_{k}^{n+1} \approx \Omega_{k}^{n}+\frac{\partial \Omega_{k}}{\partial P} P^{\prime}+\frac{\partial \Omega_{k}}{\partial e_{v}} e_{v}^{\prime}+\frac{\partial \Omega_{k}}{\partial e_{l}} e_{l}^{\prime}+\frac{\partial \Omega_{k}}{\partial X} X_{n}^{\prime}, \\
\rho_{k}^{\prime} \approx\left(\frac{\partial \rho_{k}}{\partial P}\right) P^{\prime}+\frac{\partial \rho_{k}}{\partial e_{k}} e_{k}^{\prime} .
\end{gathered}
$$

To obtain a pressure equation from Eq. (32), the unknowns $X_{n}^{\prime}$ and $e_{k}^{\prime}$ are eliminated by using the following approximation:

$$
e_{k}^{\prime} \approx e_{k}^{n}-e_{k}^{n-1} \text {, and } X_{n}^{\prime} \approx X_{n}^{n}-X_{n}^{n-1} \text {. }
$$

Then, the pressure equation becomes:

$$
D_{i} P_{i}^{\prime}+\sum_{f} D_{f} P^{\prime}{ }_{j}=D_{i}^{S}
$$


where

$$
\begin{aligned}
& D_{i}=\sum_{f} \sum_{k} \frac{\left(\alpha_{k} \rho_{k} \beta_{k}\right)_{f}}{\rho_{k, i}} \frac{A_{f}}{\mid \frac{d r_{f} \mid}{f}}-\sum_{k}\left(\frac{1}{\rho_{k}} \frac{\partial \Omega_{k}}{\partial P}\right)_{\mathrm{i}} V_{i}+\sum_{k}\left(\frac{\alpha_{k}}{\rho_{k}} \frac{\partial \rho_{k}}{\partial P}\right)_{\mathrm{i}} \frac{V_{i}}{\Delta t}, \\
& D_{f}=\sum_{k} \frac{\left(\alpha_{k} \rho_{k} \beta_{k}\right)_{f}}{\rho_{k, i}} \frac{A_{f}}{\mid \underline{d r_{f} \mid}}, \\
& D_{i}^{S}=\sum_{f} \sum_{k} \frac{1}{\rho_{k, i}}\left(\alpha_{k} \rho_{k}\right)_{f} \Psi_{k, f}^{*}+\sum_{k}\left(\frac{1}{\rho_{k}}\right)_{i} \Omega_{k i}^{n^{*}} V_{i}-\sum_{k}\left(\frac{\alpha_{k}}{\rho_{k}} \frac{\partial \rho_{k}}{\partial e_{k}} e_{{ }_{k}}\right)_{\mathrm{i}} \frac{V_{i}}{\Delta t}, \\
& \Omega_{k}^{n^{*}}=\Omega_{k}^{n}+\frac{\partial \Omega_{k}}{\partial e_{v}} e_{{ }_{v}}+\frac{\partial \Omega_{k}}{\partial e_{l}} e_{l}^{\prime}+\frac{\partial \Omega_{k}}{\partial X_{n}} X_{n}^{\prime} .
\end{aligned}
$$

The phasic density differences between neighboring cells are very small, so that the coefficient matrix of Eq. (35) is actually symmetric. Because of the symmetric pressure matrix, a fast computation is possible in comparison with the asymmetric matrix of the previous method in Section 3.1. After obtaining the new pressures, the remaining calculation sequence is the same as that in the previous method.

This scheme was found to be very useful when the number of computational cells is greater than about 10,000 [11]. But it was slightly less robust than the basic semi-implicit scheme in Section 3.1. Thus, the combined use of the two schemes was devised and tested.

\subsection{A Second-order Upwind Scheme for the Convective Terms}

In the previous versions of the CUPID code [8], the convective quantities were approximated by the firstorder upwind scheme. To enhance the numerical accuracy, a second-order upwind scheme was developed. The convective terms in the mass and energy equations are discretized as follows:

$$
\int \nabla \cdot\left(\alpha_{k} \rho_{k} \varphi_{k} \underline{u}_{k}\right) d V \approx \sum_{f}\left(\alpha_{k} \rho_{k} \varphi_{k}\right)_{f}\left(\Psi_{k}\right)_{f}^{n+1},
$$

where $\varphi_{k}$ are $1, e_{k}$, and $X_{n}$ for the k-phase continuity equation, the $\mathrm{k}$-phase energy equation, and the continuity equation for noncondensable gases, respectively. In the momentum equations, Eq. (20), two convective terms are included and are discretized as:

$$
\begin{aligned}
& \int\left[\nabla \cdot\left(\alpha_{k} \rho_{k} \underline{u}_{k} \underline{u}_{k}\right)-\underline{u}_{k} \nabla \cdot\left(\alpha_{k} \rho_{k} \underline{u}_{k}\right)\right] d V \\
& \approx \sum_{f}\left(\alpha_{k} \rho_{k} \underline{u}_{k}\right)_{f}\left(\Psi_{k}\right)_{f}^{n}-\underline{u}_{k} \sum_{f}\left(\alpha_{k} \rho_{k}\right)_{f}\left(\Psi_{k}\right)_{f}^{n} .
\end{aligned}
$$

The convective quantities in Eqs. (36) and (37), such as $\left(\alpha_{k} \rho_{k}\right)_{f},\left(\alpha_{k} \rho_{k} e_{k}\right)_{f},\left(\alpha_{g} \rho_{g} X_{n}\right)_{f}$, and $\left(\alpha_{k} \rho_{k} \underline{u}_{k}\right)_{f}$, are hereinafter denoted by $(\theta)_{f}$. These quantities are assumed to be distributed in a piecewise continuous manner, which indicates that the data on either side of a cell face may be discontinuous. The unique convective quantity at a cell face is then obtained by using an upwind method from

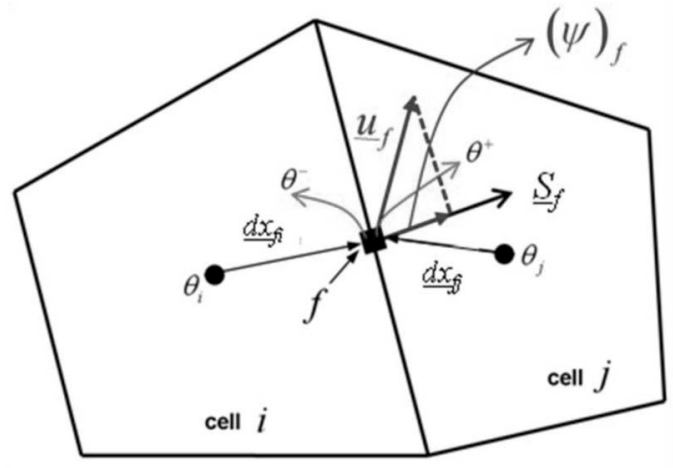

Fig. 2. Face Value Evaluation for the Upwind Scheme

two distinct convective quantities, $\theta^{-}$and $\theta^{+}$, depending on the sign of the normal velocity at a cell face, as shown in Fig. 2.

For the first-order upwind scheme, the data was assumed to be constantly distributed in a cell, and consequently two convective quantities on a face can be directly obtained from the values at the cell centers as follows:

$$
(\theta)_{f}=\left\{\begin{array}{lll}
\theta^{-}=\theta_{i} & \text { if } \quad(\Psi)_{f} \geq 0 \\
\theta^{+}=\theta_{j} & \text { if } \quad(\Psi)_{f}<0
\end{array}\right.
$$

In the second-order upwind scheme, the assumption of piecewise constant cell distribution in the first-order scheme was replaced by linear distribution. The data are extrapolated to the cell face using a Taylor series expansion about the cell center so that the convective quantities on the face are given by:

$$
(\theta)_{f}=\left\{\begin{array}{lll}
\theta^{-}=\theta_{i}+(\nabla \theta)_{i} \cdot \underline{d x}_{f i} & \text { if } \quad(\Psi)_{f} \geq 0 \\
\theta^{+}=\theta_{j}+(\nabla \theta)_{j} \cdot \underline{d x}_{f j} & \text { if } \quad(\Psi)_{f}<0
\end{array},\right.
$$

where $d x_{f k}$ represents the vector extending from the cell center to the center of the cell face. Equation (39) requires the evaluation of the gradient $\nabla \theta$ at the cell center. This gradient can be reconstructed by using a Green-Gauss theorem or by taking a least-squares approximation to the neighboring cells. Various methods have been proposed according to the contour path for the Green-Gauss reconstruction or the support of the stencil for the leastsquare reconstruction. Among those, Frink's method, originally developed on tetrahedral cells [21], was modified to evaluate the gradients on arbitrary polyhedral cells, in which the data are interpolated to the nodes first and the gradient is then evaluated using the Green-Gauss theorem along the path of a cell. The gradient at cell centers in a polyhedral cell is expressed as:

$$
(\nabla \theta)_{i}=\frac{1}{V} \sum_{f} \bar{\theta}_{f} \underline{S}_{f}
$$


where

$$
\bar{\theta}_{f}=\sum_{i=1}^{n_{p}} \theta_{n, i} / n_{p}
$$

represents a cell face value calculated by the interpolation of the face node values and $n_{p}$ denotes the number of nodes on the face. The node value $\theta_{n}$ is determined by a weighted average of the surrounding cell center values, as shown in Fig. 3:

$$
\theta_{n}=\sum_{i=1}^{n_{c}} w_{i} \theta_{i} / \sum_{i=1}^{n_{c}} w_{i}
$$

where $w_{i}$ and $n_{c}$ refer to the weighting factor for a surrounding cell and the number of surrounding cells adjacent to the node $\mathrm{n}$, respectively. The weighting factor was determined by using the pseudo-Laplacian method, which is fully second-order accurate [21]. Since Frink's reconstruction has two interpolation procedures, one from cell centers to a node and the other the Green-Gauss integration for the gradient evaluation, this method increases the support of the stencil and consequently smoothes the computed gradient for a highly distorted cell, thus enhancing the stability of the overall scheme.

The first-order upwind scheme in Eq. (38) can obtain stable solutions without non-physical oscillations in the presence of discontinuities, due to the monotonicity properties on arbitrary polygonal control volumes [22]. However, it is well known that high-order numerical schemes produce spurious oscillations in the vicinity of discontinuities, which can lead to numerical instabilities and unbounded solutions. To stabilize the numerical solutions of the second-order scheme for two-phase flows in which discontinuities might be present in convective quantities, a slope limiter, $\Phi$, was applied to Eq. (39):

$$
(\theta)_{f}=\left\{\begin{array}{lll}
\theta^{-}=\theta_{i}+\Phi_{i}(\nabla \theta)_{i} \cdot \underline{d x}_{f i} & \text { if }(\Psi)_{f} \geq 0 \\
\theta^{+}=\theta_{j}+\Phi_{j}(\nabla \theta)_{j} \cdot \underline{d x}_{f j} & \text { if } \quad(\Psi)_{f}<0
\end{array} .\right.
$$

Two multi-dimensional slope limiters of Barth and Jesperson [22] and Venkatakrishnan [23] have been

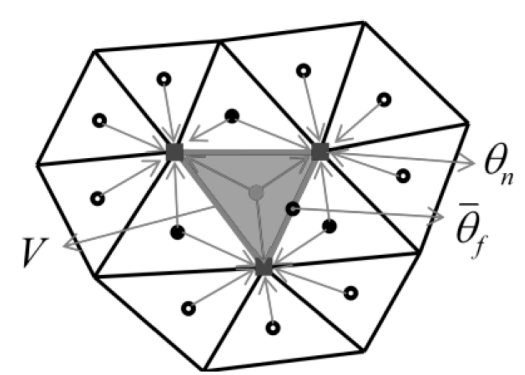

Fig. 3. Frink's Pseudo-Laplacian Weighting Method widely used in the context of unstructured finite volume frameworks. Barth and Jesperson's limiter was designed to satisfy the monotonicity principle by imposing strict restrictions for reconstructed variables not to exceed the maximum and minimum of neighboring cell values. However, it was shown to stall the convergence to a steady state due to the use of non-differentiable functions, such as max and min. To improve the convergence characteristics, Venkatakrishnan developed a new limiter by using differentiable functions at the expense of monotonicity. The limiter of Venkatakrishnan has a user-defined parameter that severely influences the convergence and oscillation-removal capability. This makes it difficult for the limiter to be applied for general-purpose solvers. Due to this unfavorable aspect of Venkatakrishnan's limiter, the limiter of Barth and Jesperson is optionally used:

$$
\Phi_{i}=\min \left(\phi_{1}, \phi_{2}, \ldots, \phi_{n_{f, i}}\right),
$$

where $n_{f, i}$ is the number of faces at a cell $i$,

$$
\begin{aligned}
& \phi_{j}= \begin{cases}\min \left(1, \Delta_{\max , i} / \Delta_{j}\right), & \text { if } \Delta_{j}>0 \\
\min \left(1, \Delta_{\min , i} / \Delta_{j}\right), & \text { if } \Delta_{j}<0, \\
1, & \text { if } \Delta_{j}=0\end{cases} \\
& \Delta_{\max , i}=\max \left(\theta_{1}, \theta_{2}, \ldots \theta_{n_{f j}}, \theta_{i}\right)-\theta_{i}, \\
& \Delta_{\min , i}=\min \left(\theta_{1}, \theta_{2}, \ldots \theta_{n_{f,},}, \theta_{i}\right)-\theta_{i}, \\
& \Delta_{j}=\left(\bar{\theta}_{f}\right)_{j}-\theta_{i} .
\end{aligned}
$$

The convective quantities should be carefully calculated, especially when $\alpha_{k}$ is very small. For example, $\left(\alpha_{k} \rho_{k}\right)_{f}$ and $\left(\alpha_{k} \rho_{k} e_{k}\right)_{f}$ obtained by Eq. (42) should satisfy the following restriction:

$$
\min \left(e_{k, i}, e_{k, j}\right) \leq\left(\alpha_{k} \rho_{k} e_{k}\right)_{f} /\left(\alpha_{k} \rho_{k}\right)_{f} \leq \max \left(e_{k, i}, e_{k, j}\right) .
$$

If the above restriction is not satisfied, the convected internal energy can be extremely small or great, leading to unphysical results. Therefore, to avoid any unexpected difficulties, the slope limiter was set to zero if $\varepsilon_{1} \leq \alpha_{k} \leq \varepsilon_{2}$, where $\varepsilon_{1}$ and $\varepsilon_{2}$ are set to $10^{-6}$ and $10^{-3}$, respectively.

\subsection{A New Scheme for the Pressure Gradient Calculation}

In the previous versions of the CUPID code $[8,9]$, the pressure gradient at a cell center was evaluated by using the Green-Gauss reconstruction method:

$$
\nabla P_{0}=\frac{1}{V_{0}} \sum_{f} P_{f} \underline{n}_{f} \underline{S}_{f}
$$

where the subscript $f$ indicates the faces of the cell 0 . In the case of a two-dimensional mesh, the pressure at the 
face $f$ was determined by:

$$
P_{f}=\frac{w_{0} P_{0}+w_{1} P_{1}}{w_{0}+w_{1}}
$$

where $P_{1}$ : the pressure at the cell 1 , which is connected to the cell 0 via the face $f$,

$$
\begin{aligned}
& w_{0}=\frac{1}{\left\|\underline{x}_{f}-\underline{x}_{0}\right\|}, \\
& w_{1}=\frac{1}{\left\|\underline{x}_{f}-\underline{x}_{1}\right\|} .
\end{aligned}
$$

This method, i.e., the Green-Gauss reconstruction with inverse-distance weighting, has second-order accuracy on structured meshes, but may have some error for skewed unstructured meshes. To overcome this problem, Frink's reconstruction was adopted in the new version of CUPID; the cell face pressure was calculated by the interpolation of the face node pressures:

$$
P_{f}=\frac{1}{2}\left(P_{a}+P_{b}\right)
$$

where $P_{a}$ and $P_{b}$ are the pressure at the nodes $a$ and $b$, consisting of the face $f$. The pressure at a node is obtained by the method in Eq. (41). Frink's reconstruction provides second-order accuracy on both structured and unstructured meshes.

The reconstruction of the pressure gradient at a boundary cell is somewhat different from that at inner cells because of the interpolation error of the pressure at the boundary face. For example, by using the Green-Gauss reconstruction method, the pressure gradient at a boundary cell can be underestimated if the pressure at the boundary face is assumed to be equal to that at the boundary cell center. To mitigate this error in the CUPID code, the pressure gradient at the boundary cell was evaluated by the leastsquare reconstruction method. In this method, the pressure surrounding a given cell 0 was assumed to be linearly distributed as:

$$
P(\underline{x})=P_{0}+\nabla P_{0} \cdot\left(\underline{x}-\underline{x}_{0}\right)
$$

Supposing that the pressures at the neighboring cell centers, $P_{j}$, satisfy Eq. (49), an over-determined system is obtained for the gradient $\nabla P_{0}$ :

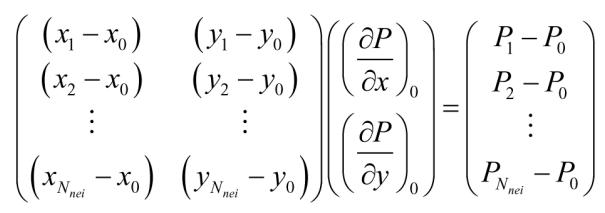

where $N_{N e i}$ denotes the number of neighboring cells for the cell 0 . The gradient at the cell center can then be determined by solving the over-determined system in a least-square sense. This method greatly enhances the accuracy of the pressure gradient at the boundary cells. However, the results of some numerical tests showed nonnegligible error at the boundary cells of skewed unstructured meshes.

Therefore, a new iterative method based on Frink's method was developed to evaluate the pressure gradient at the boundary cells. The iteration sequence is:

(a) The node pressure $P_{n}$ is determined by Eq. (41), i.e.,

$$
P_{n}=\sum_{i=1}^{n_{c}} w_{c, i} P_{c, i} / \sum_{i=1}^{n_{c}} w_{c, i}
$$

where the weighting factor is determined by using the pseudo-Laplacian method.

(b) The pressure at a cell face $P_{f}$ is calculated by using the node pressures $P_{n}$. In the case of a two-dimensional mesh, $P_{f}$ is calculated by Eq. (48).

(c) Using the Green-Gauss method, the pressure gradient at a cell center is reconstructed:

$$
\nabla P_{c}=\frac{1}{V} \sum_{f} P_{f} \underline{n}_{f} \underline{S}_{f}
$$

(d) Using the reconstructed pressure gradient in Eq. (52), the node pressure at the boundary is updated as follows:

$$
P_{n}^{\text {new }}=\frac{1}{n_{c}} \sum_{i=1}^{n_{c}}\left[P_{c, i}+\nabla P_{c, i} \cdot\left(\underline{x}_{n}-\underline{x}_{c, i}\right)\right]
$$

(e) The above steps (b) through (d) are repeated until the node pressure at the boundary converges to a certain value, i.e.,

$$
\left|P_{n}^{\text {new }}-P_{n}\right|<\varepsilon_{\text {crit }}
$$

To assess the accuracy of the above iterative methods, a simple test function in a two-dimensional space was introduced:

$$
f_{\text {test }}(x, y)=x+y \text {. }
$$

Then, the gradient of $f_{\text {test }}$ is exactly obtained:

$$
\nabla f_{\text {test }}=\underline{i}+\underline{j} .
$$

For the numerical tests, a structured grid and an unstructured 


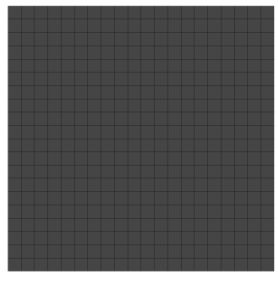

$\mathrm{GG}(\mathrm{IDW})+\mathrm{LS}(\mathrm{B})$

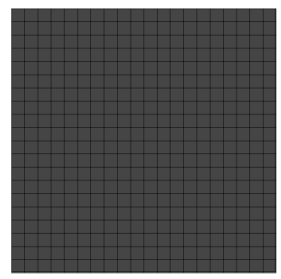

$\mathrm{GG}(\mathrm{FRK})+\mathrm{LS}(\mathrm{B})$

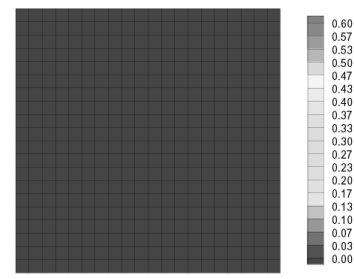

GG(FRK ITER $_{\text {I }}$

(a) Structured grid, 400 cells.

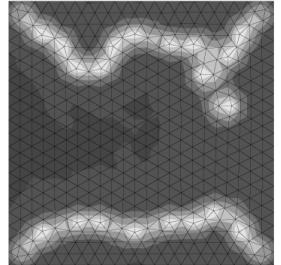

$\mathrm{GG}(\mathrm{IDW})+\mathrm{LS}(\mathrm{B})$

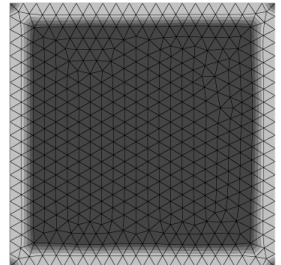

$\mathrm{GG}(\mathrm{FRK})+\mathrm{LS}(\mathrm{B})$

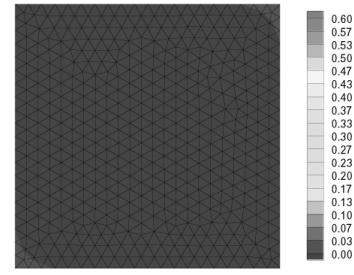

GG(FRK $\left.K_{\text {ITER }}\right)$

(b) Unstructured grid, 924 cells.

Fig. 4. Errors of the Gradients of the Test Function with Different Numerical Methods and Grids

grid, shown in Fig. 4, were used with the following three numerical methods for evaluating the gradient:

(a) GG(IDW)+LS(B): The Green-Gauss method with the inverse distance weighting for the internal cells and the least square method for the boundary cells.

(b) GG(FRK)+LS(B): The Green-Gauss method with Frink's reconstruction for the internal cells and the least square method for the boundary cells.

(c) GG(FRK ITER $)$ : The Green-Gauss method with Frink's reconstruction for the internal cells and the new iterative process for the boundary cells.

In the numerical test, the cell center values were exactly obtained from Eq. (54). Then, the gradients at the cell centers are numerically obtained by using the above three methods. The results of numerical tests are shown in Fig. 4, where the absolute value of the difference between the numerical and exact gradients is presented [24]. The errors generated in the corner boundary cells tend to propagate into the inner cells when the first two methods are used. However, the new iterative method, $\mathrm{GG}\left(\mathrm{FRK}_{\mathrm{ITER}}\right)$, yields very accurate results for both the structured and unstructured grids. The numbers of iterations were about 30 for structured meshes and about 90 for unstructured meshes, where $\varepsilon_{c r i t}$ was $10^{-7}$. This requires additional computation time, but the increment is negligible because the number of boundary cells is smaller than that of internal cells. The numerical tests using the well known cavity problem also showed that the new iterative method leads to the most accurate results for both the structured and unstructured grids [24].

\section{RESULTS OF THE PRELIMINARY ASSESSMENT}

Verification and validation [25] are the most important steps in a code development process. The verification is the assessment of the software correctness and the numerical accuracy of the solution to a given computational model. Meanwhile, the validation is the assessment of the physical accuracy of a computational model based on comparisons between computational simulations and experimental data. The CUPID code was systematically verified using various conceptual problems. The validation was under progress in a limited range. This section introduces the results of the preliminary assessment, mainly focusing on the verification.

\subsection{Conceptual Problems for the Verification}

To verify the CUPID code, various numerical tests were conducted. Table 1 summarizes the conceptual problems for the verification. These problems are divided into four groups:

(a) Steady-state flow problems were used to confirm whether the scalar equations were solved correctly. That is, the mass and energy flow rates were compared at the inlet and the exit of the computation domain, and the relative errors of the two-phase flows were found to be less than $10^{-5}$.

(b) The phase change problems were used to confirm the integrity of the numerical algorithm when a phase appears or disappears. Boiling, flashing, and condensation were tested. All these are concerned with the scalar equations [8].

(c) The phase separation problems were related to the momentum equations, in particular, the interfacial drag force and gravity. In manometric flow oscillations and dam break problems (see Section 4.6), the phases are already separated and the hydrostatic head drives the flow transient, leading to an equilibrium state. Most of the terms in the momentum equations were 
Table 1. Conceptual Problems for the Verification of the CUPID Code

\begin{tabular}{|c|c|}
\hline Problems & Remarks \\
\hline $\begin{array}{l}\text { (a) Conservation } \\
\text { - One-dimensional, steady-state liquid flow with a heat source } \\
\text { - One-dimensional, steady-state steam flow } \\
\text { - One-dimensional, steady-state two-phase flow } \\
\text { - Two-dimensional, liquid flow }\end{array}$ & \multirow{4}{*}{$\begin{array}{l}\text { Use both structured and } \\
\text { unstructured grids }\end{array}$} \\
\hline $\begin{array}{l}\text { (b) Phase change (Flow transitions) } \\
\text { - One-dimensional, boiling flow } \\
\text { - Two-dimensional, flashing flow } \\
\text { - Three-dimensional, boiling flow } \\
\text { - Cavitations with a sudden contraction and a condensation }\end{array}$ & \\
\hline $\begin{array}{l}\text { (c) Phase separation } \\
\text { - One-dimensional, air-water phase separation by gravity } \\
\text { - Two-dimensional, air-water phase separation by gravity } \\
\text { - Two-dimensional, air-water manometric flow oscillations } \\
\text { - Two-dimensional, dam break }\end{array}$ & \\
\hline$\frac{\text { (d) Treatment of noncondensable gases }}{\text { - Three-dimensional, steady-state air-water two-phase flow }}$ & \\
\hline
\end{tabular}

involved in the calculations.

(d) A three-dimensional steady-state air-water two-phase flow was simulated to confirm the continuity equations of liquid and noncondensable gas. To verify the interfacial mass transfer model under the presence of noncondensable gas, dry air $\left(X_{n}=1\right)$ was injected into the inlet of the three-dimensional duct and, then, the noncondensable gas quality $X_{n}$ was observed. $X_{n}$ should be decreased along the flow path due to the evaporation of the water vapor and its theoretical minimum can be determined by the local saturation pressure of the water. The results revealed that the CUPID code works well with non-condensable gas, as intended [8].

Through these problems, it was confirmed that the CUPID governing equations for two-phase flows were solved correctly on both structured and unstructured meshes. Some of the results are presented in the following sub-sections.

\subsection{Flow Transitions in a Vertical Pipe}

Flow transitions in a vertical pipe were simulated. The pipe is $0.1 \mathrm{~m}$ in diameter and $6 \mathrm{~m}$ in length. Slightly subcooled water at a temperature of $450.95 \mathrm{~K}$ is introduced into the bottom of the pipe. The inlet and exit pressures are maintained at 1.06 $\mathrm{MPa}$ and 1.0 $\mathrm{MPa}$, respectively, which results in an upward flow in the pipe. A volumetric heat source, of which the power is given as a function of time as shown in Fig. 5(a), is imposed on the fluid in the pipe. In this calculation, a 20 equal-length one-dimensional grid is used.
Figure 5(b) shows the volume fraction behaviors of the vapor, continuous liquid, and entrained liquid at the exit of the pipe. Up to $\sim 8 \mathrm{~s}$, the flow at the exit is a singlephase liquid flow and, then, boiling occurs due to the volumetric heating. At $\sim 12 \mathrm{~s}$, the droplet field begins to appear. Then, the flow reaches a steady state. Reducing the volumetric heat from $35 \mathrm{~s}$ results in flow transitions from a three-field flow to a single-phase liquid flow. Figure 5(b) shows that CUPID predicts a wide range of flow conditions and various flow transitions well. The conservation of the mass and energy are confirmed by comparing the steady-state inlet and exit flow conditions. This problem was calculated again using 40, 60, 120, and 240 one-dimensional meshes. Then, the axial void distributions at $30 \mathrm{~s}$ are compared in Fig 5(c), which clearly shows a mesh convergence of the CUPID code.

\subsection{A Two-dimensional Single-Phase Flow}

To verify the numerical scheme over both structured and unstructured grids, a single-phase water flow in an $\mathrm{X}$-Y plane $(0.1 \mathrm{~m} \times 0.4 \mathrm{~m})$ was simulated by using two structured and two unstructured grids. These grids are shown in Fig. 6(a). The pressures at the inlet (bottom) and the exit (top) are 1,000,020 $\mathrm{Pa}$ and 1,000,000 $\mathrm{Pa}$, respectively. The water density is $943.0 \mathrm{~kg} / \mathrm{m}^{3}$ and the viscosity is set to $0.1 \mathrm{~N} . \mathrm{s} / \mathrm{m}^{2}$ to produce a laminar flow.

A null-transient calculation was conducted to reach a steady state. Figure 6(b) compares the steady-state Ydirection velocities at $\mathrm{y}=0.3 \mathrm{~m}$. Except for the calculation with the 440 rectangular meshes, the solutions were very 


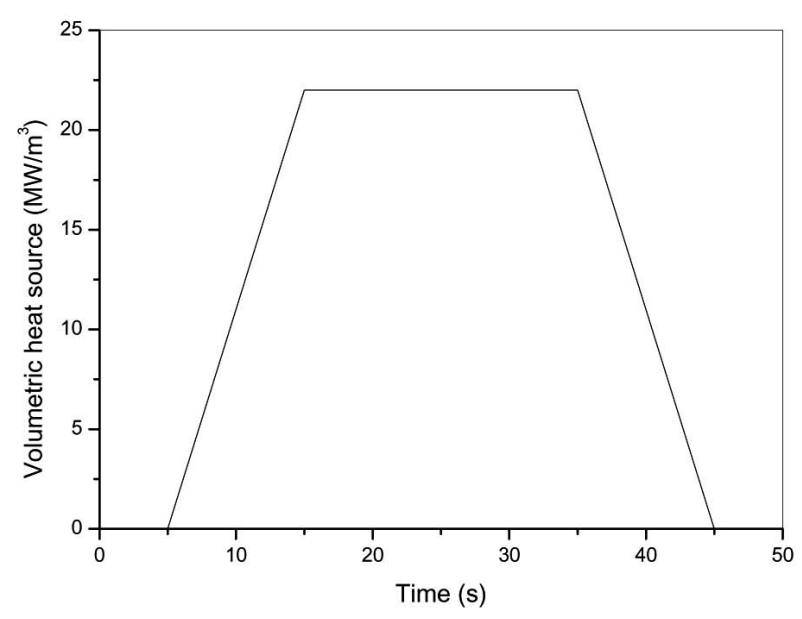

(a) Volumetric heat source vs. time

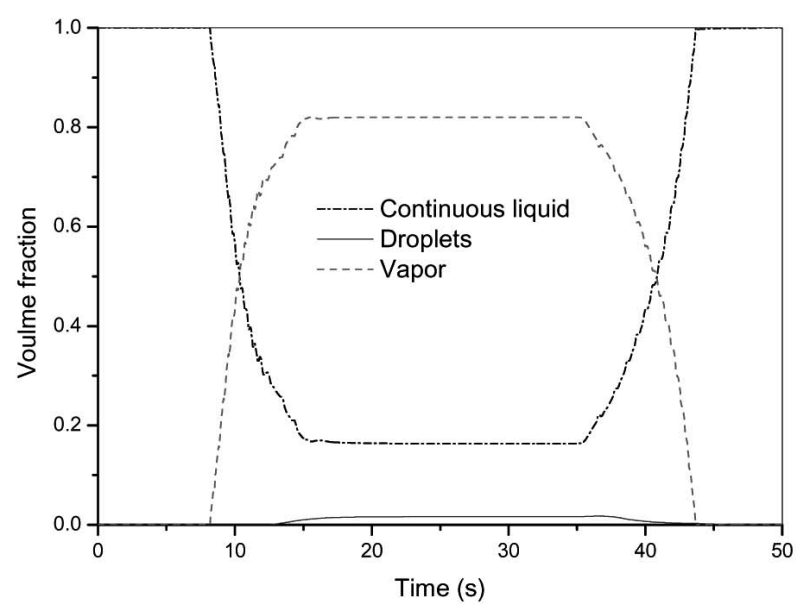

(b) Volume fraction behaviors at the exit

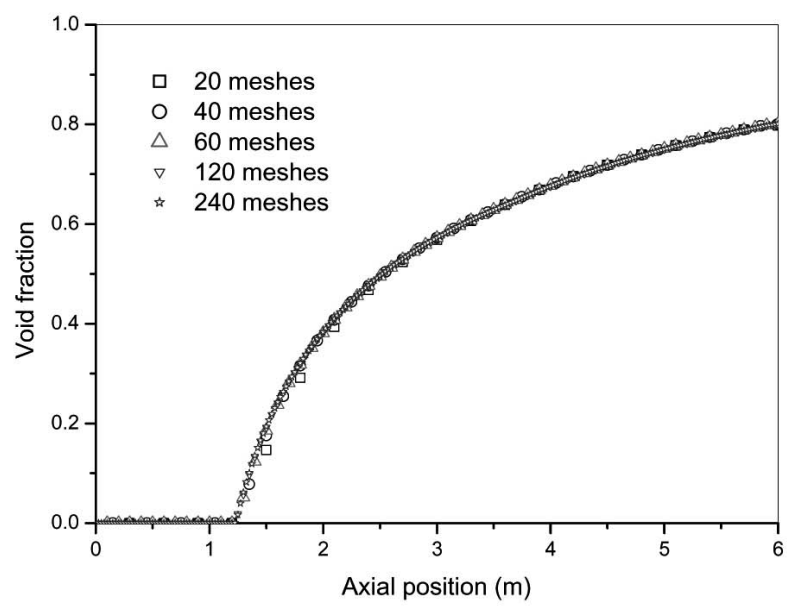

(c) Axial void distributions at $30 \mathrm{~s}$.

Fig. 5. Calculation Results of the Flow in a One-dimensional Vertical Upward Pipe

close to each other. This shows that the semi-implicit numerical scheme works for both structured and unstructured grids.

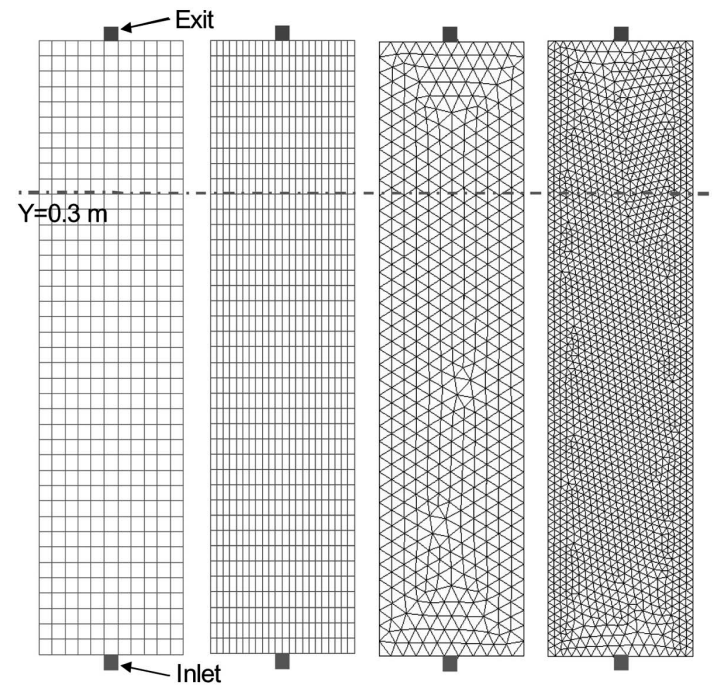

(a) Structured and unstructured grids; Rectangle 440, rectangle 880 , triangle 954 , and triangle 3,372 meshes.

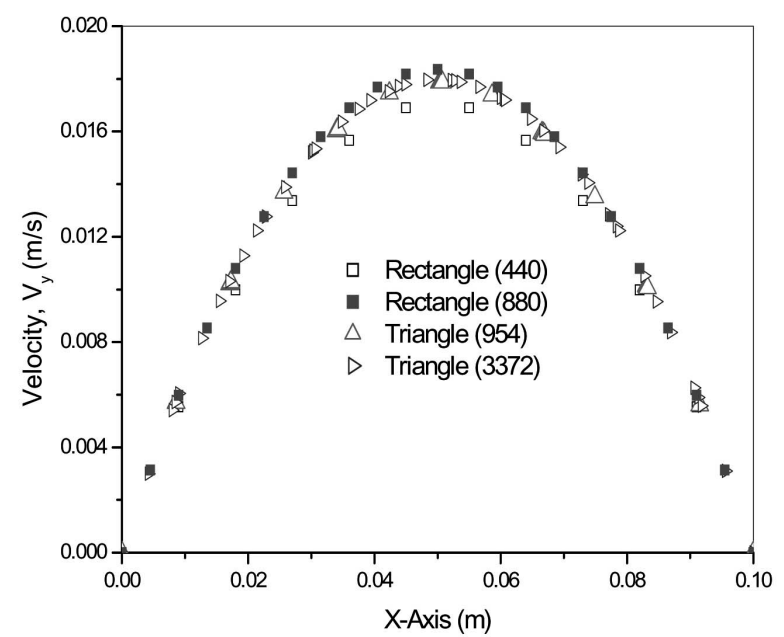

(b) Comparison of the Y-direction velocities at $y=0.3 \mathrm{~m}$.

Fig. 6. Computational Grids and Results of the TwoDimensional Single-phase Flow

\subsection{Phase Separation by Gravity in a Vertical Plane}

A numerical test for the second-order upwind scheme was conducted using the gravity-driven phase separation problem [26]. A vertical plane of $1 \mathrm{~m} \times 1 \mathrm{~m}$ was initially filled with a homogeneous two-phase mixture of air and water with a void fraction of 0.5 . The fluid motion was driven by gravity, i.e., liquid water moved downward and the air moved upward. Two steep void waves travel simultaneously from the top and bottom ends into the middle of the vertical plane, which results in the formation of a sharp interface (liquid level) after phase separation is complete. Analytical solutions for the phase separation problem were obtained with some assumptions and simplified expressions for the interfacial drag force [26]. 

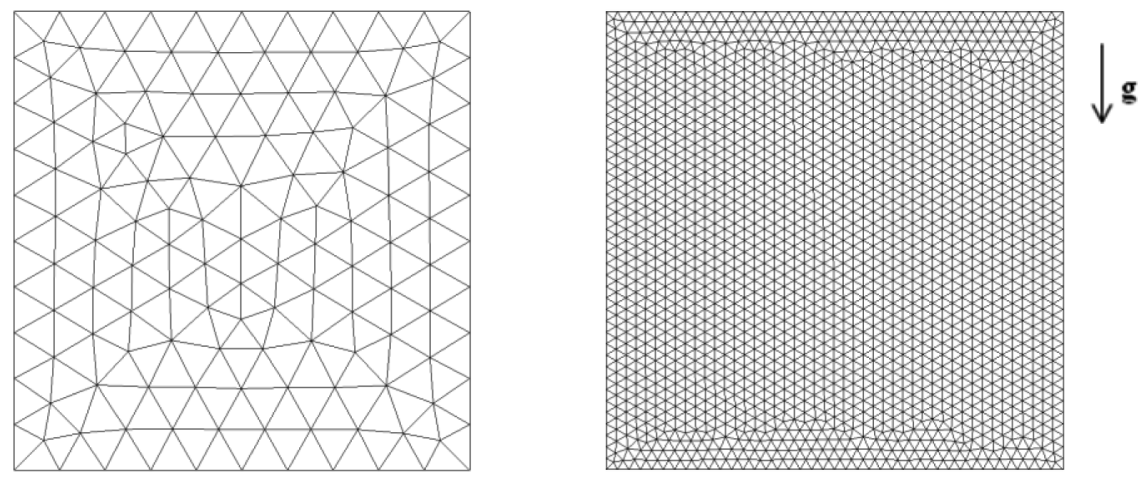

Fig. 7. Coarse and Fine Grids for the Phase Separation Problem

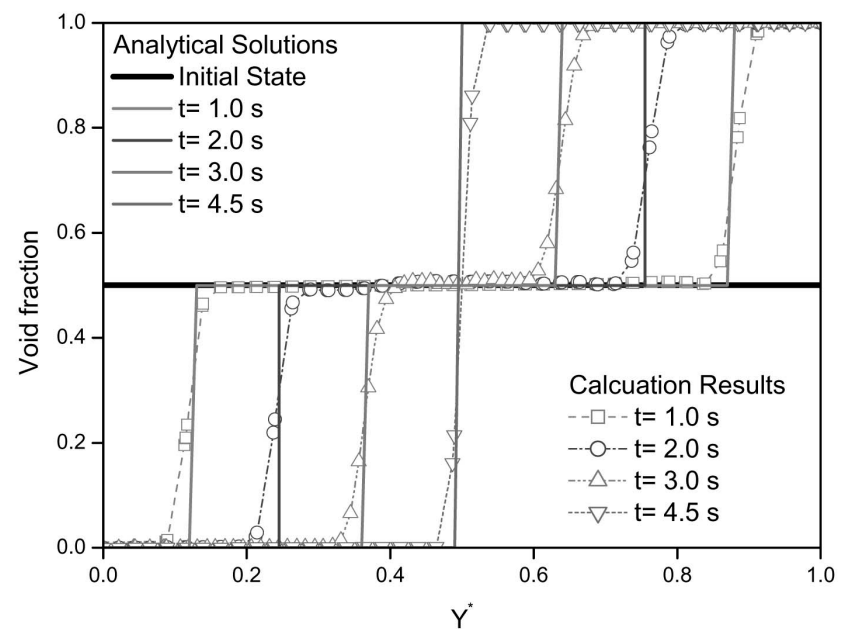

Fig. 8. Transient Void Profiles with the First-order Fine Meshes

In the CUPID calculation, the interfacial drag force was modified to be equal to that for the analytical solution.

Figure 7 shows two unstructured grids for this problem: 236 and 3702 triangular cells were used for the coarse and fine grids, respectively. Triangular meshes were used to ensure the validity of the applied reconstruction method for the unstructured meshes. It is well known that a discontinuity of convective variables between two cells can cause unphysical oscillatory numerical results. For this reason, the limiter of Barth and Jesperson was used in this calculation. Using this problem, the robustness of the second-order scheme with the limiter was also tested.

Figure 8 shows transient void profiles at $\mathrm{t}=1 \mathrm{~s}, 2 \mathrm{~s}, 3$ $\mathrm{s}$, and $4.5 \mathrm{~s}$, which were obtained with the first-order upwind fine meshes. The travelling void waves from the bottom and top of the channel are well simulated qualitatively. The four results at a fixed time of $\mathrm{t}=2.0 \mathrm{~s}$ are compared in Fig. 9; these results were calculated with the first- and second-order methods using coarse and fine meshes. It was shown that there was convergence toward the discontinuous

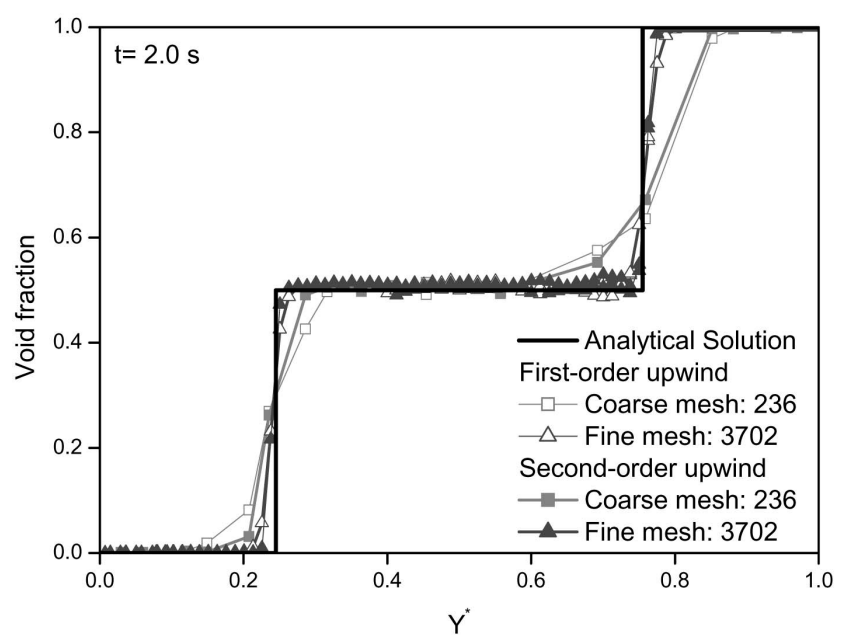

Fig. 9. Vertical Void Profiles at $\mathrm{t}=2 \mathrm{~s}$

analytical solution with an increased number of meshes. The more accurate numerical result was obtained with the second-order scheme, as can be seen in Fig. 9. Unphysical oscillatory numerical results are not observed in these calculations, which means that the Barth and Jesperson limiter, originally proposed for a single-phase compressible flow, was applicable for a two-phase flow analysis.

Because this test consists of a closed system, total water mass in the computation domain should remain constant during the transient. The relative mass error in the CUPID calculations was less than $2.1 \times 10^{-6}$, indicating that the mass was conserved very well [8].

\subsection{Cavitations with a Sudden Contraction}

Cavitations with a sudden contraction were simulated. Figure 10(a) shows the schematic of a sharp-edged contraction and its computational grid. The length and height of the left and right parts are $(0.016 \mathrm{~m} \times 0.02304 \mathrm{~m})$ and $(0.032 \mathrm{~m} \times 0.008 \mathrm{~m})$, respectively. The entire domain is modeled with 3,875 cells with the smallest size being 


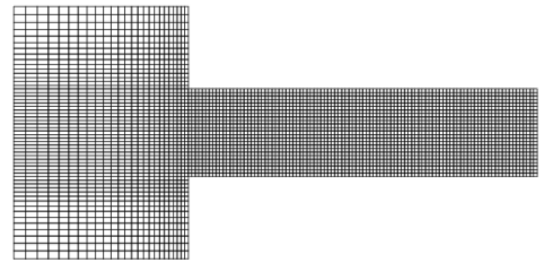

(a) Computational grid

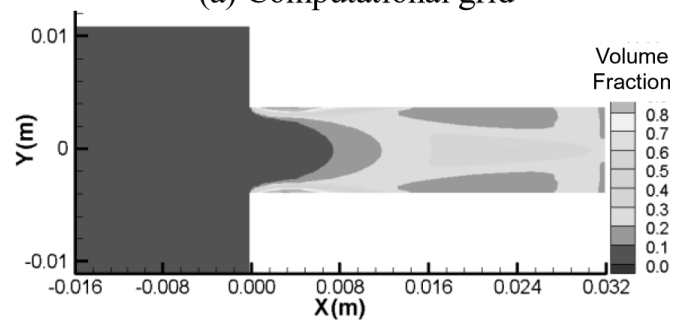

(c) CUPID: Semi-conservative

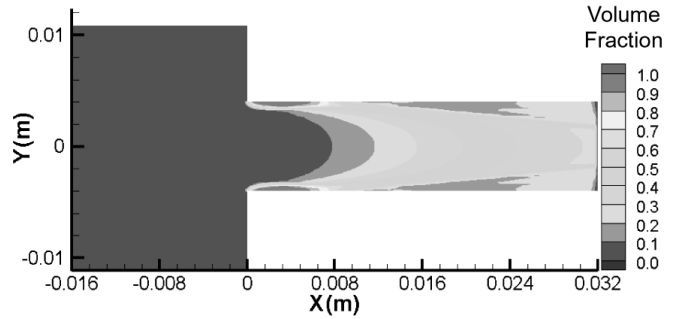

(b) FLUENT

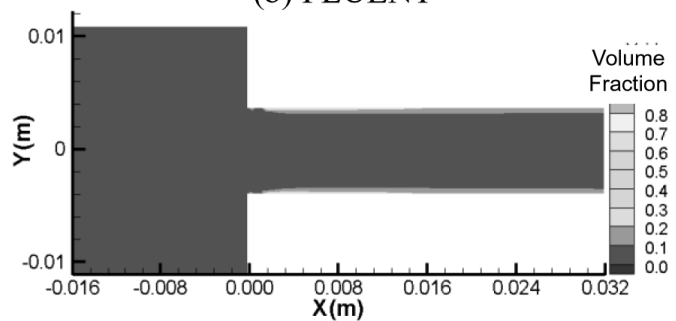

(d) CUPID: Non-conservative

Fig. 10. Computational Grid and Steady-state Void Fraction Distributions

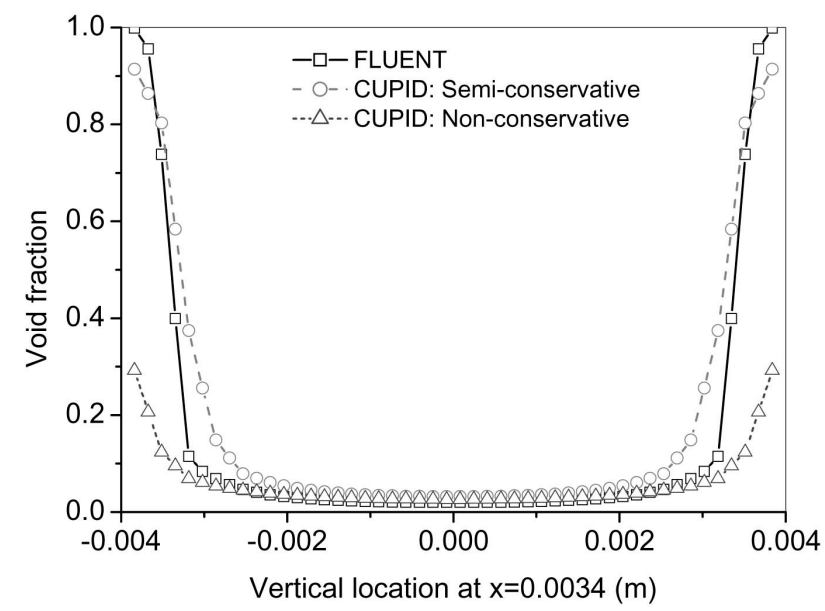

Fig. 11. Void Fraction Profile at $\mathrm{x}=0.0034 \mathrm{~m}$

$0.00032 \times 0.00032 \mathrm{~m}$. The left and the right ends were the pressure boundaries with $0.5 \mathrm{MPa}$ and $0.095 \mathrm{MPa}$, respectively. Other boundaries were walls with no-slip conditions. The water temperature was $346.1 \mathrm{~K}$, which is $28.1 \mathrm{~K}$ higher than the saturation temperature at 0.095 $\mathrm{MPa}$. These flow conditions were chosen so that cavitations would occur near the throat.

This problem was also simulated by using the FLUENT code, which has a two-fluid model with the fully conservative form of momentum equations. For this problem, the isothermal cavitation model neglecting latent heat of vaporization is used [27]. It is noted that the three-field model of the CUPID code reduces to a two-fluid model because the droplet phase does not exist in this problem.

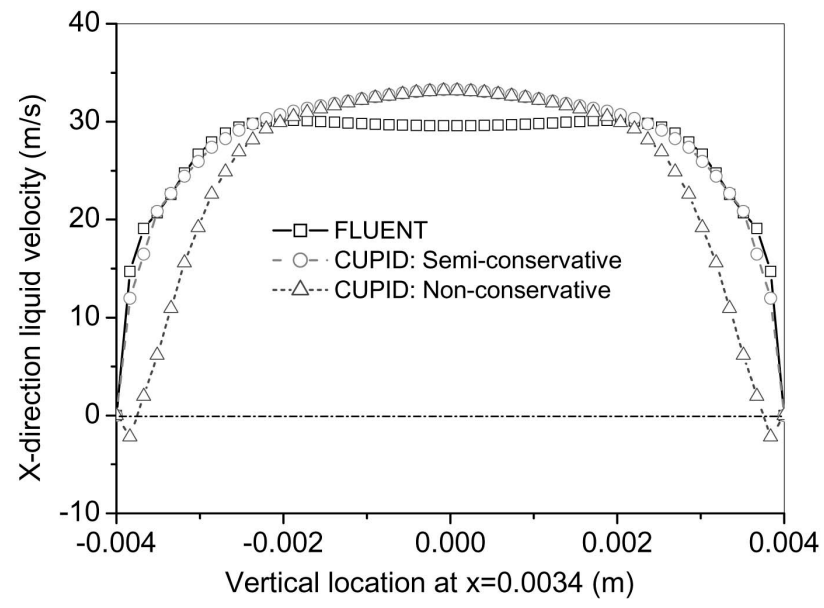

Fig. 12. Liquid Velocity $\left(V_{x}\right)$ Profile at $\mathrm{x}=0.0034 \mathrm{~m}$

Phase change by cavitations is modeled by the interfacial mass transfer term in the CUPID code. The difference in the phase change models of the two codes can entail subsequent differences in other results. Thus, a strict comparison of the two calculations is not very meaningful. The interfacial drag and virtual mass force are set equal in the CUPID and FLUENT codes. The first-order upwind scheme for the convective terms was used in the calculations to make a comparison.

Figures 10(b) through 10(d) show the steady-state void distributions of three different momentum equations: the conservative FLUENT, the semi-conservative CUPID, and the non-conservative CUPID calculations [10]. Strong cavitations occur near the orifice after flow separation at 
the sharp edge. The result of the semi-conservative form is similar to that of the FLUENT code, the void fraction near the walls increases to the maximum at $\mathrm{x}=\sim 0.0034$ $\mathrm{m}$. The void distribution after the sharp edge is spatially very heterogeneous. Maximum local void fractions of the semi-conservative CUPID and FLUENT codes are 0.91 and 1.0, respectively. Figure 10(d) shows that the void distribution with the non-conservative form is quite different from those of the semi-conservative CUPID and the conservative FLUENT codes. To clearly illustrate the differences, the vertical void and $\mathrm{x}$-direction liquid velocity profiles at $\mathrm{x}=0.0034 \mathrm{~m}$ are presented in Figs. 11 and 12, respectively. The non-conservative CUPID shows poor predictions of the void profile and the flow field after separation at the sharp edge. These results show that the semi-conservative form yields a better solution, especially for heterogeneous two-phase flows. Thus, the semiconservative form is adopted in the CUPID code.

\subsection{Dam Break}

The dam break experiment is very useful for both the verification and the validation of multi-phase flow codes. Experiments by Nagatake, et al. [28] were used for the verification of the CUPID code. The schematic of the experiment is depicted in Fig. 13. Initially, the water column was blocked by a stainless steel plate. The plate was instantaneously pulled up, and then the water behavior was recorded with a high-speed camera. Case 4 was simulated for verification; the water column width, $L$, was $5.0 \mathrm{~cm}$.

To simulate this problem, both two- and threedimensional meshes were used. However, since the two calculations yielded almost the same results, only the two-dimensional meshes with $30 \times 30$ and $60 \times 60$ cells were used thereafter. Figure 14 shows the calculated water behavior of the $30 \times 30$ cells. The free surface of the collapsed water column is well predicted qualitatively, indicating that the local flow regime concept of CUPID [12] works as intended. In about five seconds, the water reaches an equilibrium state. The main parameter in this experiment is the water front position during the transient. Figure 15 compares a dimensionless water front position, $x^{*}=x / L$, vs. a dimensionless time, $t^{*}=t(2 g / L)^{0.5}$. The calculated water front position in Fig. 15 is not continuous, but rather discrete, because the water front was assumed to reach a computational cell when $\alpha_{g}<0.99$. It can be seen that, by using fine meshes, the solution gets closer to the experimental data, and that the water fronts of the second-order computations are propagated more slowly than those of

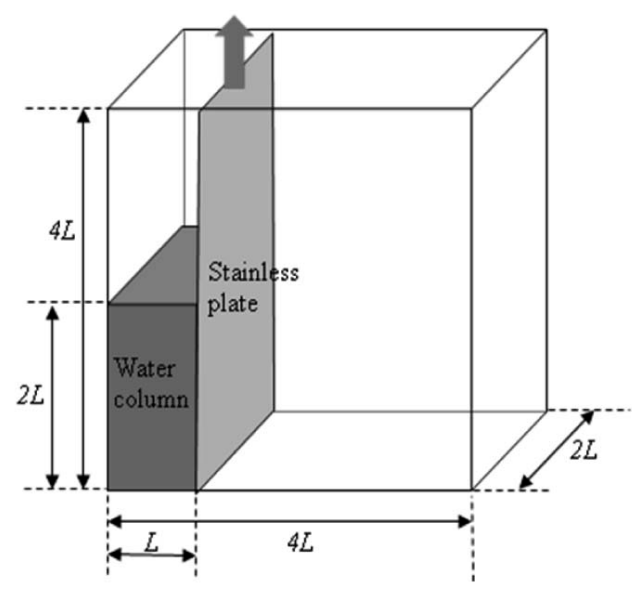

Fig. 13. Schematic of the Dam-break Experiment

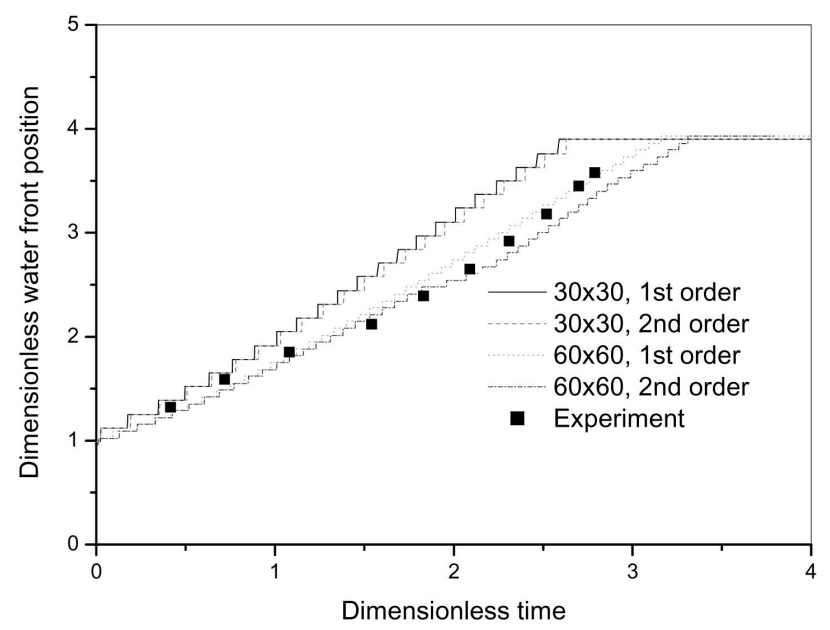

Fig. 15. Comparison of the Water Front Positions

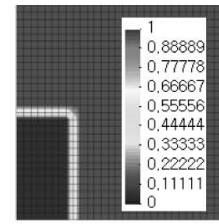

(a) $\mathrm{t}=0.0 \mathrm{~s}$

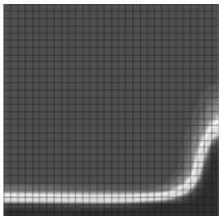

(b) $\mathrm{t}=0.3 \mathrm{~s}$

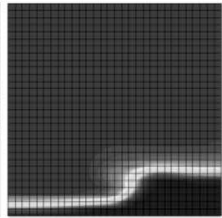

(c) $\mathrm{t}=0.45 \mathrm{~s}$

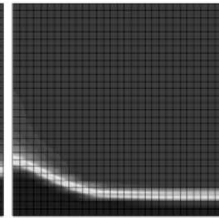

(d) $\mathrm{t}=0.75 \mathrm{~s}$

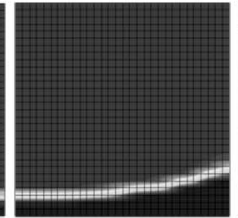

(e) $\mathrm{t}=1.9 \mathrm{~s}$

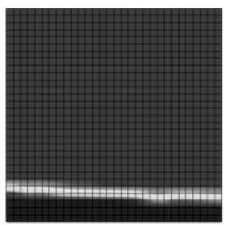

(f) $\mathrm{t}=3.15 \mathrm{~s}$

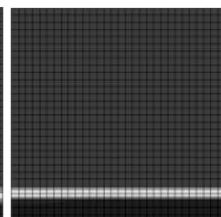

(g) $\mathrm{t}=5.5 \mathrm{~s}$

Fig. 14. Results of the CUPID Calculation: Void Distributions during the Transient 


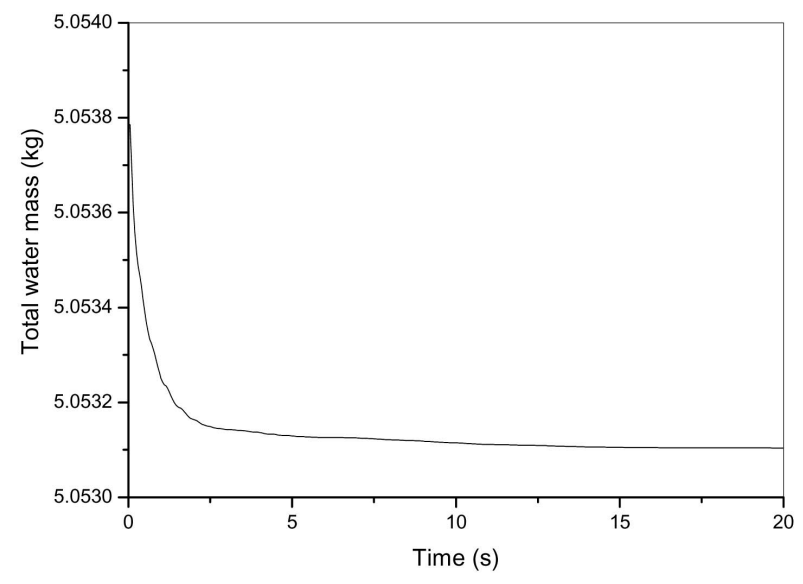

Fig. 16. Total Mass vs. Time

the first order computations, which means that the secondorder scheme is less dissipative. The second-order scheme does not always guarantee better accuracy. Accuracy is more concerned with relevant physical models than with numerical schemes.

Figure 16 shows the total mass behavior during the transient. Because the test facility is nearly a closed system in the CUPID calculation, total water mass in the computation domain should remain almost constant. However, because there was an opening on the top of the computation domain to impose a constant pressure boundary condition, this allowed for little mass flow through the boundary. Figure 16 shows that the total mass is conserved reasonably well with a maximum relative change of $1.4 \times 10^{-4}$.

\subsection{Downcomer Boiling}

An experimental facility, named DOBO, was designed to simulate the downcomer boiling phenomena that may occur in the lower downcomer region during the reflood phase of a postulated LBLOCA [15]. The DOBO consists of a test section, a steam-water separator, a condenser, a heat exchanger, a drain pump, a storage tank, an air injection and ventilation system, a pre-heater, and an injection pump. The test section has a rectangular duct geometry; its dimensions are $6.4 \mathrm{~m}$ high, $0.25 \mathrm{~m}$ deep and $0.30 \mathrm{~m}$ wide. The height and gap size are the same as those of the reference plant downcomer, but the circumference is reduced 47.08-fold. One among the four side walls of the test section incorporates 207 cartridge heaters inside it to simulate the stored energy release from the reactor vessel wall. The heated region starts $0.3 \mathrm{~m}$ above the inlet, and ends $1.0 \mathrm{~m}$ below the outlet. The major measuring parameters are the local void fraction, local gas and liquid velocities, local fluid temperature, differential pressures along the elevation, pressures, and liquid flow rate.

For the assessment of the CUPID code, an upward liquid flow test, DOBO-C2-1 Test [29], was selected. The liquid inlet flow rate and temperature are $3.2 \mathrm{~kg} / \mathrm{s}$

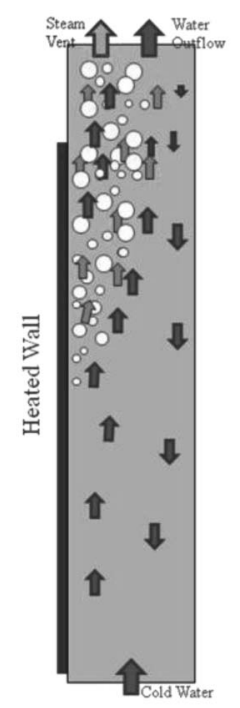

(a) The schematic

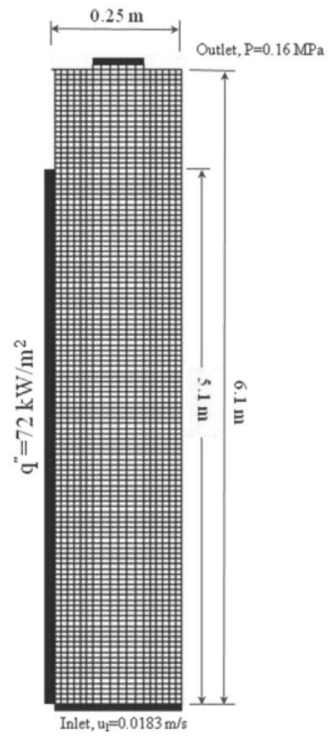

(b) Computational mesh
Fig. 17. The Schematic and the Computational Mesh of the DOBO Experiment

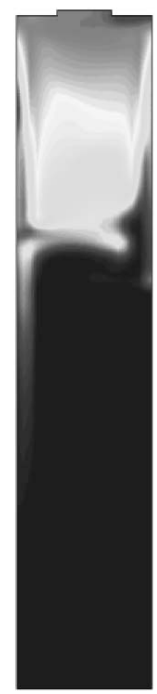

Void

fraction

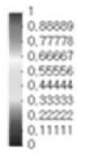

Fig. 18. Time-averaged Void Distribution

(i.e., $0.0183 \mathrm{~m} / \mathrm{s}$ at the inlet) and $111.9^{\circ} \mathrm{C}$, respectively. The heat flux from the heated wall is $72 \mathrm{~kW} / \mathrm{m}^{2}$, and the outlet pressure is maintained at $0.16 \mathrm{MPa}$. Figure $17(\mathrm{a})$ shows the schematic of the test. When bubbles are generated on the heated wall, the liquid near the wall is accelerated by the rising bubbles. Then, the liquid outside the bubble region flows transversely toward the heated wall for the mass balance. The inflow of the relatively cold water into the bubble region results in a rapid decrease of boiling; therefore, the void fraction is lowered. The cold water is heated up again along the wall and the void fraction 
bounces back followed by the liquid flow acceleration. This phenomenon is repeated, resulting in periodic generation of bubbles.

In this calculation, it was assumed that the flow behavior along the width direction is relatively negligible compared to the axial and depth directions and, hereafter, the test section was analyzed with a two-dimensional approach, in order to save computational time. The $18 \times 122$ computational mesh is shown in Fig. 17(b). Constant velocity and constant pressure boundary conditions were imposed on the inlet and outlet, respectively. A constant heat flux was applied on the heated wall.

To deal with the periodic oscillations of the flow field, the calculation was continued for a 1500 -second problem time after a quasi-steady state was achieved, and the time averaged results were used for the comparison of the computational results and those of the experiment. Figure 18 shows the time-averaged void distribution of the CUPID calculation.

Figure 19(a) compares the horizontal void fraction distributions at elevations of $3.5 \mathrm{~m}$ and $4.5 \mathrm{~m}$. The CUPID code with the default bubble size model over-predicted the void fraction. To investigate which models caused this discrepancy, a series of sensitivity studies were performed with various models for the bubble diameter, interfacial drag and heat transfer models. It was found that the computational results are highly sensitive in the bubble size model. The bubble size strongly affects the interfacial drag and non-drag forces, as well as the interfacial area. In this regard, a new bubble size model was suggested so that the bubble size can increase gradually with the void fraction up to 0.3 ; the minimum bubble diameter was restricted by the TRAC-M model [30,31]. The changes of the bubble size model are summarized as follows [12]: (a) The default model

$$
\begin{aligned}
& D_{b, \text { Yoneda }}=10.06\left(\frac{10^{5}}{P}\right)^{0.098} \sqrt{\frac{\sigma}{g\left(\rho_{l}-\rho_{g}\right)}} \cdot\left[\min \left(\alpha_{g}, \alpha_{B}\right)\right]^{0.35} \\
& \text { where } \alpha_{B}=0.118 .
\end{aligned}
$$

(b) The modified model

$$
\begin{aligned}
& D_{b}=\max \left(D_{b, \text { Yoneda }}, D_{\text {TRAC }}\right), \\
& \text { where } D_{\text {TRAC }}=2 \sqrt{\frac{\sigma}{g\left(\rho_{l}-\rho_{g}\right)}} \text { and } \alpha_{B}=0.3 .
\end{aligned}
$$

Figure 19(b) shows the calculation results with the modified bubble diameter model. The void distributions at $3.53 \mathrm{~m}$ and $4.5 \mathrm{~m}$ were enhanced remarkably. The overall trend of the velocity profile at $3.5 \mathrm{~m}$ was captured reasonably as well. In the churn flow region, however, there are still considerable discrepancies of the velocity profile; in particular, the downward liquid of the calculation result near the wall region was not observed in the test results [12]. This assessment result showed that a further improvement of the physical models of the two-fluid model is required for the churn-flow region. In a future study, this issue will be investigated in order to improve the performance of the CUPID code for multi-dimensional two-phase flow analysis.

It was revealed through this assessment that a more systematic approach is needed for the improvement of the physical models for multi-dimensional two-phase flow, because there are very complicated interactions between the momentum source terms. For example, increasing the interfacial drag coefficient leads to a decrease in the relative

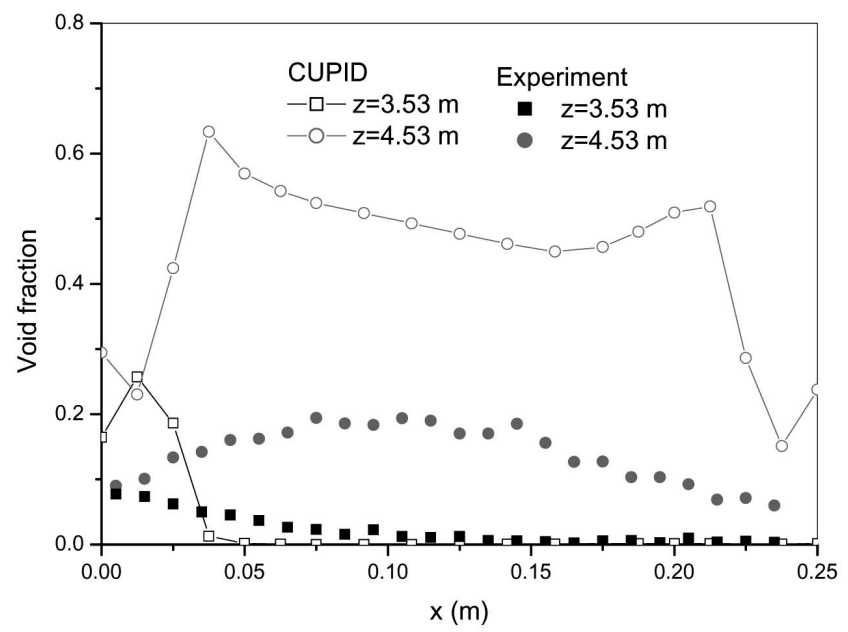

(a) The default bubble size model

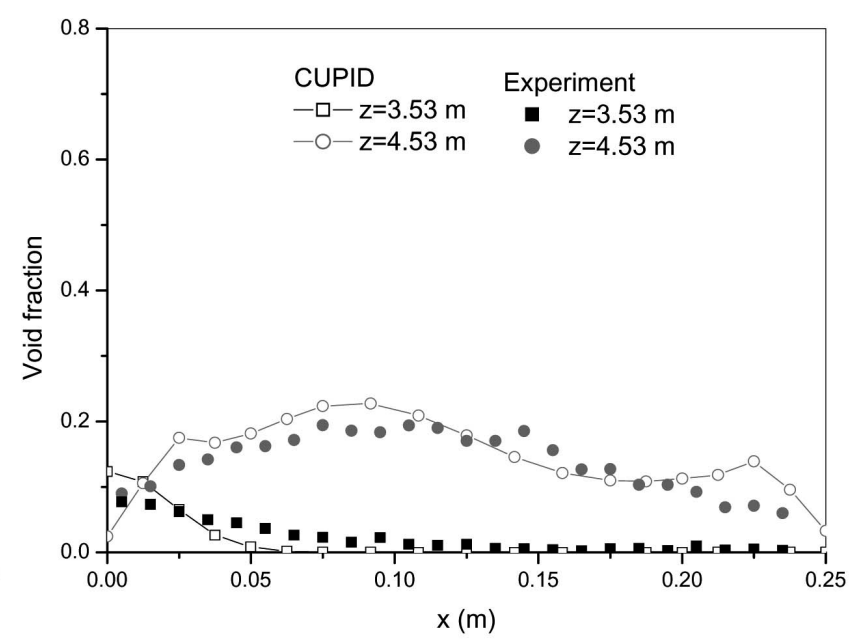

(b) The modified bubble size model

Fig. 19. Comparison of the Calculated and the Measured Horizontal Void Distributions with Different Bubble Size Models 
velocity and an increase in the void fraction. This again results in a change in the lift force, leading to a change in the transverse void distribution. Then, the turbulent dispersion force is also changed in these interactions. All these, in turn, affect the interfacial drag force.

\section{CONCLUSIONS}

The three-dimensional thermal hydraulics code, CUPID, was developed for realistic simulation of transient two-phase flows in nuclear reactor components. In the CUPID code, a two-fluid three-field model was adopted for two-phase flows. The semi-implicit numerical method of the REALP5 code was extended for application to unstructured grids and was improved for enhanced accuracy and faster running. To verify the CUPID code, a set of conceptual problems and some experiments were simulated. The calculations were carried out for both structured and unstructured grids including one-, two-, and three-dimensional meshes. It was shown that the numerical scheme was accurate and very robust for the predictions of single- and two-phase flows, phase changes and flow transitions due to boiling and flashing, phase separations, and air-water two-phase flows. Conservation of the mass and energy was also confirmed, and the momentum equations worked as designed. Thus it can be said that the CUPID code was successfully verified. The results of the downcomer boiling calculations show that the CUPID code is very promising, but a systematic approach for the validation and improvement of the physical models is needed.

\section{ACKNOWLEDGEMENTS}

This work was supported by the Nuclear Research \& Development Program of the NRF (National Research Foundation), through a grant funded by the MEST (Ministry of Education, Science and Technology) of the Korean government (Grant code: M20702040002-08M020400210).

\section{NOMENCLATURE}

$\begin{array}{ll}A & \text { Surface area } \\ e_{k} & \text { Internal energy of k-phase } \\ g & \text { Gravitational acceleration } \\ H_{i k} & \text { Volumetric interfacial heat transfer coefficient } \\ M_{i k} & \text { Interfacial momentum transfer } \\ \underline{n}_{f} & \text { Outward face normal vector } \\ P & \text { Pressure } \\ Q_{i k} & \text { Interfacial heat transfer to k-phase } \\ S_{D E} & \text { Droplet de-entrainment rate per volume } \\ S_{E} & \text { Droplet entrainment rate per volume } \\ \underline{S}_{f} & \text { Surface vector } \\ t & \text { Time } \\ T & \text { Temperature } \\ \underline{u}_{k} & \text { Phasic velocity, } \underline{u}_{k}=u_{k} \underline{i}+v_{k} \underline{j}+w_{k} \underline{k}\end{array}$

$$
\begin{array}{ll}
\frac{u_{k i}}{x} & \text { Interface velocity of k-phase } \\
\bar{X}_{n} & \text { Position vector } \\
& \text { Noncondensable gas quality }
\end{array}
$$

\section{Greek Symbols}

$\begin{array}{ll}\alpha_{k} & \text { Volume fraction of k-phase, where } a_{v}+a_{l}+a_{d}=1 \\ \Delta t & \text { Time step size, } t^{n+1}-t^{n} \\ \delta \varphi & \varphi^{n+1}-\varphi^{n} \text { where } \varphi=X_{n}, e_{v}, e_{l}, \alpha_{v}, \alpha_{d}, \text { or } P \\ \Gamma_{v} & \text { Vapor generation rate per volume } \\ \Psi_{k} & \text { Phasic volume flow } \\ \rho & \text { Density } \\ \tau_{k} & \text { Viscous shear stress } \\ \tau_{k}^{T} & \text { Turbulent shear stress } \\ \Omega_{k} & \text { Inter-phase mass transfer rate per volume }\end{array}$

\section{Subscripts}

$d \quad$ Entrained liquid (droplets)

$f \quad$ Saturated water or cell face

$g \quad$ Saturated steam

$i \quad$ Interface

$k \quad$ k-field, i.e., continuous liquid, entrained liquid, or vapor

$l \quad$ Continuous liquid

$n \quad$ Noncondensable gases

$s \quad$ Steam

sat Saturated

$v \quad$ Vapor (Steam and noncondensable gas mixture)

$w \quad$ Wall

\section{Superscripts}

$n \quad$ Old time step

$n+1 \quad$ New time step

\section{REFERENCES}

[ 1 ] G. Yadigaroglu, M. Andreani, J. Dreier, and P. Coddinggton, Trends and needs in experimentation and numerical simulation for LWR safety, Nuclear Engineering and Design, 221, pp. 205-223 (2003).

[2] M. Scheuerer et al., Evaluation of computational fluid dynamic methods for reactor safety analysis (ECORA), Nuclear Engineering and Design, 235, pp. 359-368 (2005).

[ 3 ] D. Bestion et al., Extension of CFD codes to two-phase flow safety problems, NEA/SEN/SIN/AMA (2006)2, OECD Nuclear Energy Agency.

[4] B.L. Smith et al., Assessment of CFD for nuclear reactor safety problems. Final for approval by CSNI, OECD Nuclear Energy Agency, 2007.

[ 5 ] U. Bieder and E. Graffard, Qualification of the CFD code Trio_U for full scale reactor applications, Nuclear Engineering and Design, 238(3), pp. 671-679 (2008).

[6] G. Yadigaroglu, Computational fluid dynamics for nuclear applications: From CFD to multi-scale CMFD, Nuclear Engineering and Design, 235(2-4), pp. 153-164 (2005).

[7] A. Guelfi et al., NEPTUNE: a new software platform for advanced nuclear thermal hydraulics, Nuclear Science and Engineering, 156(3), pp. 281-324 (2007).

[ 8 ] J.J. Jeong, H.Y. Yoon, I.K. Park, H.K. Cho, and J. Kim, A 
Semi-implicit numerical scheme for transient two-phase flows on unstructured grids, Nuclear Engineering and Design, 238, pp. 3403-3412 (2008).

[ 9 ] I.K. Park, H.K. Cho, H.Y. Yoon, and J.J. Jeong, Numerical effects of the semi-conservative form of momentum equations for multi-dimensional two-phase flows, Nuclear Engineering and Design, 239, pp. 2365-2371 (2009).

[10] I.K. Park, H.K. Cho, H.Y. Yoon, and J.J. Jeong, Implementations of non-drag interfacial forces into the CUPID code, Proceedings of ICAPP 2009, Tokyo, Japan, May 10-14, 2009.

[11] H.Y. Yoon, J.J. Jeong, I.K. Park, H.K. Cho, and H. Lee, An improved semi-implicit numerical scheme for transient three-dimensional two-phase flow analysis, The $13^{\text {th }}$ International Topical Meeting on Nuclear Reactor Thermal Hydraulics, Kanazawa, Japan, Sep. 27- Oct. 2, 2009.

[12] H.K. Cho, B.J. Yun, I.K. Park, and J.J. Jeong, Computational analysis of downcomer boiling phenomena using a component thermal hydraulic analysis code CUPID, to be presented at the 18th International Conference on Nuclear Engineering, Xi'an, China, May 17-21, 2010.

[13] The RELAP5-3D Code Development Team, RELAP5-3D code manual volume I: Code structure, system models and solution methods, Idaho National Engineering and Environmental Laboratory (2001).

[14] J.J. Jeong, I. Dor, and D. Bestion, Improvement and assessment of the CATHARE2 three-dimensional module compared with the UPTF downcomer test 7, Nuclear Technology, 117, pp. 267-280 (1997).

[15] B.J. Yun, D.J. Euh, and C.H. Song, Investigation of the downcomer boiling phenomena during the reflood phase of a postulated large-break LOCA in the APR1400, Nuclear Technology, 156, pp. 56-68 (2006).

[16] J.J. Jeong, K.S. Ha, B.D. Chung, and W.J. Lee, Development of a multi-dimensional thermal-hydraulic system code, MARS 1.3.1, Annals of Nuclear Energy, 26(18), pp. 16111642 (1999).

[17] C. Frepoli, J.H. Mahaffy, and K. Ohkawa, Notes on the implementation of a fully-implicit numerical scheme for a two-phase three-field flow model, Nuclear Engineering and Design, 225, pp. 191-217 (2003).

[18] D.R. Liles and W.H. Reed, A semi-implicit method for two- phase fluid dynamics, J. Comput. Phys., 26, pp. 390-407 (1978).

[19] H.Y. Yoon et al., An unstructured SMAC algorithm for thermal non-equilibrium two-phase flows, Int. Communications in Heat and Mass Transfer, 36, pp. 16-24 (2009).

[20] C.M. Rhie, and W.L. Chow, Numerical study of the turbulent flow past an airfoil with trailing edge separation, $A I A A$ Journal 21(11), pp. 1525-1532 (1983).

[21] N.T. Frink, Recent progress toward a three-dimensional unstructured Navier-Stokes flow solver, AIAA Paper 940061 (1994).

[22] T.J. Barth and D.C. Jesperson, The design and application of upwind schemes on unstructured meshes, AIAA Paper 89-0366 (1989).

[23] V. Venkatakrishnan, Convergence to steady state solutions of the Euler equations on unstructured grids with limiters, Journal of Computational Physics, 118, pp. 120-130 (1995).

[24] H.D. Lee, A new method for the reconstruction of the pressure gradient for the CUPID code, CTHA-2009-005, Korea Atomic Energy Research Institute (2009).

[25] W.L. Oberkampf and T.G. Trucano "Verification and validation benchmarks," Nuclear Engineering and Design, 238, 716-743 (2008).

[26] H. Staedtke, Gas dynamic aspects of two-phase flow, WileyVCH Verlag GmbH \& Co. KGaA Weinheim, pp. 143-147 (2006).

[27] FLUENT 6.3 user's guide, Fluent Inc. (2006).

[28] T. Nagatake, Z. Kawara, and T. Kunugi, Establishment of experimental data base on dam-breaking problem for validating interface tracking methods, NTHAS6: Sixth Japan-Korea Symposium on Nuclear Thermal Hydraulics and Safety, Okinawa, Japan, Nov. 24-27, 2008.

[29] D.J. Euh et al., Experimental characterization of co-current subcooled boiling phenomena in a rectangular geometry, to be presented at HEFAT2010, 7th International Conference on Heat Transfer, Fluid Mechanics and Thermodynamics, 19-21 July, Antalya, Turkey, 2010.

[30] J.W. Spore et al., TRAC-M/FORTRAN 90 (VERSION 3.0) THEORY MANUAL, LA-UR-00-910, Los Alamos National Laboratory, 2000.

[31] M. Ishii, Personal communication to R. Nelson, Los Alamos National Laboratory, 1987. 